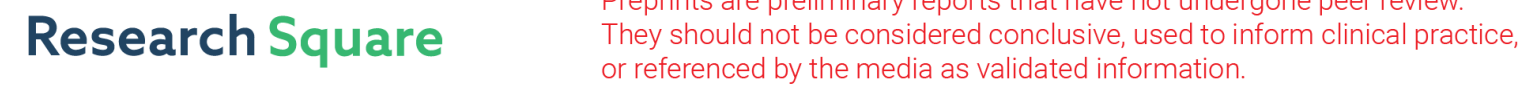 \\ Preprints are preliminary reports that have not undergone peer review.
}

\section{Real-world Experience of Nintedanib Therapy in Idiopathic- inflammatory-myopathy-related Interstitial Lung Disease: Efficacy and Tolerability}

\author{
Junyu Liang \\ Zhejiang University School of Medicine First Affiliated Hospital \\ Heng Cao \\ Zhejiang University School of Medicine First Affiliated Hospital \\ Yang Yang \\ Zhejiang University School of Medicine First Affiliated Hospital \\ Yini Ke \\ Zhejiang University School of Medicine First Affiliated Hospital \\ Chuanyin Sun \\ Zhejiang University School of Medicine First Affiliated Hospital

\section{Lihuan Yue} \\ Zhejiang University School of Medicine First Affiliated Hospital \\ Jin Lin ( $\nabla$ linjinzju@zju.edu.cn ) \\ Zhejiang Province
}

\section{Research article}

Keywords: Nintedanib, Interstitial lung disease, Myositis, anti-MDA5 antibody, anti-PM-Scl75 antibody

Posted Date: August 10th, 2020

DOI: https://doi.org/10.21203/rs.3.rs-52262/v1

License: (c) (i) This work is licensed under a Creative Commons Attribution 4.0 International License. Read Full License 


\section{Abstract \\ Background}

Interstitial lung disease (ILD) is a common and frequently fatal extra-muscular complication in patients with idiopathic inflammatory myopathy (IIM), and is refractory to the conventional immunosuppressive medications. Nintedanib has previously been proven to be effective and tolerable in idiopathic pulmonary fibrosis, systemic-sclerosis-related ILD, etc. However, the efficacy and safety of nintedanib in idiopathicinflammatory-myopathy-related ILD (IIM-ILD) remain unknown. The purpose of this study was to initially explore the efficacy and tolerability of nintedanib in IIM-ILD patients.

\section{Methods}

A real-world analysis was conducted to explore the efficacy and tolerability of nintedanib in IIM-ILD patients who regularly received outpatient visit or hospitalization from January 2018 to October 2019 in one medical center. The primary end point was occurrence of rapid progression of interstitial lung disease (RP-ILD) in the follow-up. And time to death from any cause, complication of pulmonary infection and difference in immunosuppressive regimen were taken as secondary end points in this study. Adverse events were descriptively recorded.

\section{Results}

22 patients receiving nintedanib therapy and 82 patients under conventional medications were included. After propensity score matching, the primary comparison revealed that better survival $(P=0.036)$ and prominently less RP-ILD $(P=0.031)$ in patients with nintedanib therapy. Logistic regression analysis identified that disease activity $(P=0.032)$, anti-PM-Scl75 antibody $(P=0.027)$ and nintedanib therapy $(P=0.023$, OR value $=0.063)$ were significantly correlated with RP-ILD. Cox proportional hazards regression analysis demonstrated that disease activity $(P$ $=0.007)$, anti-MDA5 antibody $(P=0.004)$ and nintedanib therapy $(P=0.027$, HR value $=0.190)$ were significantly associated with survival of IIMILD patients. Similar results can also be seen in analyses before propensity score matching. In the 22 patients with nintedanib therapy, diarrhea was the most common adverse event (54.5\%) and hepatic insufficiency contributed to most dosage reduction (50\%) or therapy discontinuation (50\%).

\section{Conclusion}

Nintedanib therapy might reduce incidence of RP-ILD and improve survival in IIM-ILD patients. Anti-PM-ScI75 and anti-MDA5 antibodies could predict RP-ILD and survival respectively. In addition to the most frequent diarrhea, hepatic insufficiency was closely related to dosage reduction or therapy discontinuation.

\section{Trial registration:}

ISRCTN.com, ISRCTN 10507540. Retrospectively registered.

\section{Introduction}

Idiopathic inflammatory myopathy (IIM) comprises a group of autoimmune diseases featuring systemic inflammation of the proximal skeletal muscles and/or the skin [1, 2] Dermatomyositis (DM) and polymyositis (PM) are two classical subtypes of IIM, whereas amyopathic dermatomyositis (ADM) is a newly recognized subgroup of DM with typical skin rash of DM and slight/absent muscular impairment[1-3]. IIM contributes to elevated morbidity and mortality rate due to severe muscle weakness as well as multiple organ involvement including the esophagus, heart, and the lungs in particular [4]. Interstitial lung disease (ILD) is the most common extra-muscular target in IIM patients, with a reported prevalence of $78 \%$, and is found in $65 \%$ of newly diagnosed IIM cases [5-7]. Idiopathic-inflammatory-myopathy-related ILD (IIM-ILD) is frequently aggressive and refractory to conventional therapies including glucocorticoids and immunosuppressive drugs [7-9]. Meanwhile rapid progression of interstitial lung disease (RP-ILD) has constituted a major cause of mortality in IIM patients [10,11]. Under such circumstance, it would be necessary to take a broader view and search for more potent therapeutic agent for IIM-ILD.

Nintedanib, formerly known as BIBF 1120, is a small-molecule tyrosine kinase inhibitor that targets vascular endothelial growth factor (VEGF) receptor-1 3, platelet-derived growth factor (PDGF) receptor- $\alpha$ and $-\beta$, fibroblast growth factor (FGF) receptor-1 3, etc. [12] Initially developed as a cancer therapeutic, it was subsequently recognized as an antifibrotic agent $[13,14]$. Preceding researches in animal models with manifestations of fibrosing ILDs like idiopathic pulmonary fibrosis (IPF), systemic-sclerosis-related ILD (Ssc-ILD), rheumatoid-arthritis-related 
ILD (RA-ILD), hypersensitivity pneumonitis and silicosis have uncovered that nintedanib has anti-fibrotic effect regardless of the trigger for the ILD pathology [15]. And the therapeutic value of nintedanib has been demonstrated in different subgroups of ILD via TOMORROW, INPULSIS-1, INPULSIS-2, SENSCIS and INBUILD trials as well as several real-world analyses [16-20]. However, the effect and safety of nintedanib in IIM-ILD remain an enigma to researchers and physicians. It is thus necessary to fill in the gaps by sorting out the efficacy and tolerability of nintedanib in IIM-ILD patients.

In the past two years, compassionate use of nintedanib, in addition to the immunosuppressive medications, was initiated for a few IIM-ILD patients after clarifying diagnosis of IIM-ILD and acquiring written informed consent. Other IIM-ILD patients who disagree in the same period received conventional immunosuppressive therapies. To acquire an initial impression on the efficacy and tolerability of nintedanib in IIM-ILD, we retrospectively reviewed the medical records of all IIM-ILD patients who were regularly treated and followed-up in the outpatient and inpatient department of our center from January 2018 to October 2019, and performed a real-world analysis to figure out the therapeutic value, dosage regimen and profile of adverse events of nintedanib in IIM-ILD.

\section{Patients And Methods}

\section{Patients}

After acquiring the approval (Reference Number: 2020 - 200, 2018 - 224) from the Clinical Research Ethics Committee of the First Affiliated Hospital, College of Medicine, Zhejiang University (FAHZJU) and written informed consent to utilize and publish clinical data from all patients involved, in accordance with the Declaration of Helsinki, we retrieved medical records of adult patients who were regularly treated and visited in the outpatient or inpatient department of FAHZJU with the diagnosis of IIM-ILD from January 2018 to October 2019. The inclusion criteria of this study were: 1) age over 18 years old; 2) the diagnosis of DM, PM or ADM satisfied the 2017 ACR/EULAR classification criteria [21]; 3) regular outpatient visit or hospitalization in FAHZJU; Exclusion criteria were: 1) clarified overlap syndromes with other connective tissue diseases (CTDs); 2) outpatient visit or hospitalization for reasons unrelated to myositis and its complications, such as fracture, pregnancy, acquired immunodeficiency syndrome and cataract, etc. due to lack of demanded medical records for this study; 3 ) preceding use of nintedanib, or preceding/present use of pirfenidone; 4) loss to follow-up without death from any cause within 6 months after initial outpatient visit or hospitalization.

\section{Methods}

Medical records of all enrolled patients were retrospectively collected by reviewing the electronic medical record (EMR) system of FAHZJU. Data including demographic information, course of disease, duration of diagnosis delay, disease activity assessment, clinical manifestations or complications, preceding comorbidities, harmful hobbies, radiological/laboratory findings, lung function testing, immunosuppressive medications as well as compassionate use of nintedanib were acquired and analyzed. To obtain the survival, etc. of patients involved, Patients were followed from the initial outpatient visit or hospitalization until the end of follow-up. For patients who perished during hospitalization, their dates of death were clearly documented in the EMR system. For patients who were discharged, a routine return visit was arranged 2 weeks after discharge. In addition to the regular inpatient or outpatient visits, a concise telephone interview was performed 3 months after discharge, and at an annual frequency afterwards. The end of follow-up could be owing to death from any cause, loss to follow-up, or closure of follow-up for the purpose of this study (April 30th, 2020). For patients receiving compassionate nintedanib therapy, initial dosage, dosage adjustment, discontinuation and duration of nintedanib as well as adverse events were also collected from the EMR and follow-up records.

Disease activity of IIM was routinely assessed utilizing the Myositis Disease Activity Assessment Visual Analogue Scales (MYOACT) at the initial outpatient visit or within the first week of hospitalization [22]. ILD and its progression were evaluated by experienced radiologists using high-resolution computed tomography (HRCT). Cases manifested as usual interstitial pneumonia (UIP) pattern were recognized based on their HRCT appearance: the presence of basal-dominant reticular opacities and predominantly basal and subpleural distribution of honeycomb lesions, with multiple equal-sized cystic lesions of 2 to $10 \mathrm{~mm}$ diameter with a thick wall [23]. A subset of patients with RP-ILD was defined as those presenting with progressive dyspnea and progressive hypoxemia, a worsening of interstitial change on the chest radiograph within 1 month after the initial visit or onset of respiratory symptoms [24-27]. Infection, pulmonary infection in particular, was a common complication in IIM patients and a key contributor to unfavorable outcome. Identification of pulmonary infection relied on International Statistical Classification of Diseases, 10th revision (ICD-10)-coded discharge diagnosis of community-acquired pneumonia (CAP), hospital-acquired pneumonia (HAP), pulmonary fungal infection or pulmonary infection. Responsible pathogens were recognized based on repeated cultures/smears of bronchoalveolar lavage fluid (BALF) or sputum before related treatment.

To acquire the positivity of 12 myositis-specific antibodies (MSAs, anti-MDA5, anti-TIF1Y, anti-Jo-1, anti-OJ, anti-PL-7, anti-PL-12, anti-EJ, antiMi-2a, anti-Mi-2 $\beta$, anti-NXP2, anti-SRP and anti-SAE1) and 4 myositits-associated antibodies (MAAs, anti-Ro-52, anti-PM-Scl75, anti-PM-Scl100, 
anti-Ku) of IIM patients, sera from these patients were tested using the EUROLINE Autoimmune Inflammatory Myopathies 16 Ag (IgG) commercial line blot assay (Euroimmun, Lübeck, Germany) encompassing a membrane strip with the 16 autoantigens in light of the manufacturer's instructions. To fulfill the testing, the detected serum samples were pipetted into an incubation channel containing the test strips and incubated for 30 minutes, after which the strips were washed, and an enzyme conjugate was pipetted into the incubation channel. 30 minutes later, the strips were washed again and a substrate was pipetted into the channel. Finally, the strips were rinsed with distilled water. And EUROLineScan (Euroimmun) was used to interpret the positivity of the assays.

The primary end point was occurrence of RP-ILD in the follow-up. And time to death from any cause was taken as key secondary end point in this study. Since complication and progression of ILD usually lead to more potent immunosuppressive therapy and the subsequent complication of pulmonary infection in clinical practice, other prespecified secondary end points included complication of pulmonary infection and difference in immunosuppressive regimen. Due to the initial lack of follow-up lung function testing and recent impact (suspension of lung function testing) of Corona virus disease 2019 (COVID-19) pandemic, decline of forced vital capacity (FVC) was not listed as an end point in this study.

Comparisons were made between patients receiving compassionate nintedanib therapy and patients under conventional medications to clarify differences in clinical symptoms, complications, radiological/laboratory findings, survival, etc. Propensity score matching was applied to alleviate patient selection bias and to adjust for confounding factors that might interfere with the comparison between the two groups. The unadjusted effects of nintedanib on RP-ILD, time to death from any cause, complication of infection and immunosuppressive regimen were hereby initially probed into. Afterwards Cox proportional hazards regression analysis was performed to identify the influence of clinical factors, nintedanib in particular, on survival of these patients. Logistic regression analysis was used to identify the effect of nintedanib or other clinical factors on other end points (occurrence of RP-ILD, complication of pulmonary infection and difference in immunosuppressive regimen) with statistical significance. In patients receiving compassionate use of nintedanib, initial dosage, dosage adjustment, discontinuation and duration of nintedanib therapy as well as adverse events were documented and compared with those reported in IPF or Ssc-ILD.

\section{Statistical analysis}

Statistical analysis was performed using SPSS 22.0 (Chicago, IL, USA) and R 3.6.1. The normality of continuous variables was tested by the Kolmogorov-Smirnov goodness-of-fit model. Continuous variables were expressed as mean \pm SD if normally distributed and median (quartiles) if skewed. Ordinal categorical variables were as well shown as median (quartiles). Unordered categorical variables were presented as numbers and percentages. In comparison between patients receiving compassionate nintedanib therapy and patients under conventional medications, independent sample t-test was used to compare normally distributed continuous variables. And Mann-Whitney $U$ test was applied to compare skewed continuous variables or ordinal categorical variables. Chi-square test and Fisher's exact test were utilized to compare unordered categorical variables.

Propensity score matching was performed to adjust for age, sex as well as statistically different factors in comparison. The propensity scores were calculated using logistic regression analysis with Statistical Package for SPSS 22.0 (Chicago, IL, USA). Due to the small sample size of this study, a 1:4 matching was performed without replacement, using nearest neighbor matching and a caliper width of 0.5 of the standard deviation

Survival in groups with compassionate nintedanib therapy or conventional treatment was estimated using the Kaplan-Meier method, and differences between groups were compared using the log-rank test. Univariate and multivariate Cox proportional hazards regression analyses were subsequently adopted to identify the influence of nintedanib and other factors on the time to death from any cause. Univariate and multivariate logistic regression analyses were used to unveil whether nintedanib and other factors impacted the left end point (the risk of RPILD, complication of pulmonary infection or immunosuppressive regimen) with statistical significance. Explanatory factors with $\mathrm{P}<0$. 1 in the univariate analysis would be entered into the multivariate analysis. P values in comparisons and univariate analyses were adjusted by false discovery rate (FDR) correction, using p.adjust function in R.3.6.1, to obtain adjusted P values (P-adjusted). All tests were two-sided, and P < 0.05 was considered statistically significant. Adverse events were presented descriptively.

\section{Results}

A total of 104 IIM-ILD patients who satisfied the inclusion \& exclusion criteria from January 2018 to October 2019 were finally incorporated into this study, encompassing 67 with DM, 24 with PM and 13 with ADM. 40 were males (38.5\%) and the medium age of all the patients included was $58.0(49.3,65.0)$ years old. The medium follow-up time was $9.8(7.1,15.0)$ months. 22 of them received compassionate nintedanib therapy as well as immunosuppressive therapy after clarifying diagnosis and acquiring written informed consent (Supplementary table 1 ). The other 82 patients who disagree were under conventional immunosuppressive medications. (Fig. 1) An unadjusted comparison between patients receiving compassionate nintedanib therapy and patients under conventional medications identified that patients with nintedanib therapy had

Page $4 / 23$ 
lower percent-predicted diffusing capacity of the lung for carbon monoxide (DLCO\%, $P=0.006)$, but exhibited better survival ( $P=0.043$, Fig. 2 ). Besides, only $4.5 \%$ of patients with nintedanib therapy suffered from RP-ILD, which was much lower than that in patients under conventional medications (22.0\%). After adjusting for age, sex and DLCO\% using propensity score matching, lower incidence of RP-ILD (P = 0.031) as well as better survival $(P=0.036$, Fig. 3 ) was identified in patiens receiving nintedanib therapy. However, the significance vanished after FDR correction. No statistical significance was found in complication of pulmonary infection or immunosuppressive regimen between the two groups. (Table 1 )

To further evaluate the effect of nintedanib in risk of RP-ILD, univariate and multivariate logistic regression analyses were utilized to adjust for other clinical factors. In univariate analysis, MYOACT score $(P=0.038)$, positivity of anti-PM-Scl75 antibody $(P=0.075)$ as well as nintedanib therapy $(P=0.093)$ were found to be associated with risk of RP-ILD at the level of $P<0.1$. The following multivariate logistic regression analysis revealed that MYOACT score $(P=0.023)$, positivity of anti-PM-Scl75 antibody $(P=0.044)$ and nintedanib therapy $(P=0.048,0 R$ value $=0.107)$ were significantly correlated with risk of RP-ILD in patients with IIM-ILD. Similar results were identified after propensity score matching. MYOACT score $(P=0.032)$, positivity of anti-PM-Scl75 antibody $(P=0.027)$ and nintedanib therapy $(P=0.023,0 R$ value $=0.063)$ were still significantly associated with RP-ILD in these patients (Table 2) 
Table 2

Univariate and multivariate logistic regression analysis of RP-ILD in IIM-ILD patients

\begin{tabular}{|c|c|c|c|c|c|c|c|c|}
\hline \multirow[b]{2}{*}{ Factors } & \multicolumn{4}{|c|}{ Before propensity score matching } & \multicolumn{4}{|c|}{ After propensity score matching } \\
\hline & Nintedanib(22) & $\begin{array}{l}\text { Non- } \\
\text { nintedanib(82) }\end{array}$ & $\begin{array}{l}\mathrm{P} \\
\text { value }\end{array}$ & $\begin{array}{l}\text { P- } \\
\text { adjusted }\end{array}$ & Nintedanib(22) & $\begin{array}{l}\text { Non } \\
\text { nintedanib(58) }\end{array}$ & $\begin{array}{l}P \\
\text { value }\end{array}$ & $\begin{array}{l}\mathrm{P} \text { - } \\
\text { adjusted }\end{array}$ \\
\hline $\operatorname{Age}(\mathrm{y})$ & 55.5(47.3,64.3) & $58.5(49.8,65.0)$ & 0.459 & 0.961 & $55.5(47.3,64.3)$ & $57.5(48.5,63.5)$ & 0.678 & 1.000 \\
\hline Sex(male/female) & $9 / 13$ & $31 / 51$ & 0.790 & 1.000 & $9 / 13$ & $26 / 32$ & 0.752 & 1.000 \\
\hline Course of disease(m) & $4.5(2.0,10.3)$ & $3.5(1.0,9.0)$ & 0.500 & 0.961 & $4.5(2.0,10.3)$ & $4.0(1.0,12.5)$ & 0.991 & 1.000 \\
\hline $\begin{array}{l}\text { Duration of diagnosis } \\
\text { delay }(m)\end{array}$ & $2.0(1.0,5.0)$ & $2.5(1.0,5.0)$ & 0.725 & 1.000 & $2.0(1.0,5.0)$ & $3.0(1.0,7.3)$ & 0.713 & 1.000 \\
\hline \multicolumn{9}{|c|}{ Clinical manifestations or complications } \\
\hline Dysphagia & $3(13.6 \%)$ & $12(14.6 \%)$ & 1.000 & 1.000 & $3(13.6 \%)$ & $4(6.9 \%)$ & 0.386 & 1.000 \\
\hline Dysarthria & $1(4.5 \%)$ & $7(8.5 \%)$ & 1.000 & 1.000 & $1(4.5 \%)$ & $3(5.2 \%)$ & 1.000 & 1.000 \\
\hline $\begin{array}{l}\text { Respiratory muscle } \\
\text { involvement }\end{array}$ & $0(0.0 \%)$ & $4(4.9 \%)$ & 0.576 & 0.961 & $0(0.0 \%)$ & $3(5.2 \%)$ & 0.557 & 1.000 \\
\hline Cardiac involvement & $2(9.1 \%)$ & $2(2.4 \%)$ & 0.196 & 0.816 & $2(9.1 \%)$ & $2(3.4 \%)$ & 0.303 & 1.000 \\
\hline $\begin{array}{l}\text { Gastrointestinal } \\
\text { hemorrhage }\end{array}$ & $3(13.6 \%)$ & $5(6.1 \%)$ & 0.361 & 0.961 & $3(13.6 \%)$ & $4(6.9 \%)$ & 0.386 & 1.000 \\
\hline $\begin{array}{l}\text { Pulmonary bacterial } \\
\text { infection }\end{array}$ & $4(18.2 \%)$ & $22(26.8 \%)$ & 0.406 & 0.961 & $4(18.2 \%)$ & $15(25.9 \%)$ & 0.471 & 1.000 \\
\hline $\begin{array}{l}\text { Pulmonary fungal } \\
\text { infection }\end{array}$ & $0(0.0 \%)$ & $4(4.9 \%)$ & 0.576 & 0.961 & $0(0.0 \%)$ & $1(1.7 \%)$ & 1.000 & 1.000 \\
\hline Carcinoma & $3(13.6 \%)$ & $14(17.1 \%)$ & 1.000 & 1.000 & $3(13.6 \%)$ & $10(17.2 \%)$ & 1.000 & 1.000 \\
\hline UIP pattem & $6(27.3 \%)$ & $18(22.0 \%)$ & 0.599 & 0.961 & $6(27.3 \%)$ & $17(29.3 \%)$ & 0.857 & 1.000 \\
\hline Pneumomediastinum & $1(4.5 \%)$ & $4(4.9 \%)$ & 1.000 & 1.000 & $1(4.5 \%)$ & $3(5.2 \%)$ & 1.000 & 1.000 \\
\hline Survivala $^{\mathrm{a}}(\mathrm{m})$ & $12.8(8.4,19.6)$ & $9.6(6.3,14.2)$ & 0.043 & 0.816 & $12.8(8.4,19.6)$ & $9.8(6.3,14.7)$ & 0.036 & 0.936 \\
\hline RP-ILD & $1(4.5 \%)$ & $18(22.0 \%)$ & 0.069 & 0.816 & $1(4.5 \%)$ & $16(27.6 \%)$ & 0.031 & 0.936 \\
\hline \multicolumn{9}{|l|}{ Disease activity } \\
\hline MYOACT score & $8.0(7.0,9.3)$ & $8.0(6.0,10.0)$ & 0.776 & 1.000 & $8 \cdot 0(7 \cdot 0,9.3)$ & $8.0(6.0,10.3)$ & 0.729 & 1.000 \\
\hline \multicolumn{9}{|l|}{ Lung function testing } \\
\hline FVC\%(\%) & $67.2 \pm 16.6$ & $73.7 \pm 18.2$ & 0.134 & 0.816 & $67.2 \pm 16.6$ & $71.5 \pm 18.1$ & 0.333 & 1.000 \\
\hline TLC(L) & $3.6 \pm 1.2$ & $3.8 \pm 0.9$ & 0.598 & 0.961 & $3.6 \pm 1.2$ & $3.7 \pm 0.9$ & 0.955 & 1.000 \\
\hline FEV1\%(\%) & $69.3 \pm 17.0$ & $75.8 \pm 18.8$ & 0.145 & 0.816 & $69.3 \pm 17.0$ & $72.7 \pm 17.6$ & 0.429 & 1.000 \\
\hline FEV1/FVC & $0.8(0.8,0.9)$ & $0.8(0.8,0.9)$ & 0.759 & 1.000 & $0.8(0.8,0.9)$ & $0.8(0.8,0.9)$ & 0.910 & 1.000 \\
\hline DLCO\%(\%) & $51.7 \pm 13.7$ & $63.4 \pm 18.0$ & 0.006 & 0.312 & $51.7 \pm 13.7$ & $56.1 \pm 14.5$ & 0.220 & 1.000 \\
\hline
\end{tabular}

RP-ILD: Rapid progression of interstitial lung disease; IIM-ILD: Idiopathic-inflammatory-myopathy-related interstitial lung disease; y: years; m: months; UIP pattern: Usual interstitial pneumonia pattern; MYOACT: Myositis Disease Activity Assessment Visual Analogue Scales; FVC\%: Percent-predicted forced vital capacity; TLC: Total lung capacity; L: Litre; FEV1\%: Percent-predicted forced expiratory volume in 1 second; FEV1/FVC: Ratio of FEV1 over FVC; DLCO\%: Percent-predicted diffusing capacity of the lung for carbon monoxide; DMARDs: Disease-modifying anti-rheumatic drugs; IVIG: Intravenous immunoglobulin; IIM: Idiopathic inflammatory myopathy; DM: dermatomyositis; PM: Polymyositis; ADM: Amyopathic dermatomyositis. 
Myositis-specific antibodies \& Myositis-associated antibodies

\begin{tabular}{|c|c|c|c|c|c|c|c|c|}
\hline Anti-MDA5 & $6(27.3 \%)$ & $16(19.5 \%)$ & 0.557 & 0.961 & $6(27.3 \%)$ & $10(17.2 \%)$ & 0.355 & 1.000 \\
\hline Anti-PL-7 & $2(9.1 \%)$ & $10(12.2 \%)$ & 1.000 & 1.000 & $2(9.1 \%)$ & $10(17.2 \%)$ & 0.495 & 1.000 \\
\hline Anti-PL-12 & $1(4.5 \%)$ & $2(2.4 \%)$ & 0.514 & 0.961 & $1(4.5 \%)$ & $0(0.0 \%)$ & 0.275 & 1.000 \\
\hline Anti-EJ & $3(13.6 \%)$ & $4(4.9 \%)$ & 0.162 & 0.816 & $3(13.6 \%)$ & $4(6.9 \%)$ & 0.386 & 1.000 \\
\hline Anti-Ro-52 & $11(50.0 \%)$ & $36(43.9 \%)$ & 0.610 & 0.961 & $11(50.0 \%)$ & $26(44.8 \%)$ & 0.679 & 1.000 \\
\hline Anti-Jo-1 & $2(9.1 \%)$ & 14(17.1\%) & 0.513 & 0.961 & $2(9.1 \%)$ & $13(22.4 \%)$ & 0.215 & 1.000 \\
\hline Anti-OJ & $1(4.5 \%)$ & $2(2.4 \%)$ & 0.514 & 0.961 & $1(4.5 \%)$ & $1(1.7 \%)$ & 0.477 & 1.000 \\
\hline Anti-TIF1y & $0(0.0 \%)$ & 11(13.4\%) & 0.115 & 0.816 & $0(0.0 \%)$ & $8(13.8 \%)$ & 0.099 & 1.000 \\
\hline Anti-Ku & $0(0.0 \%)$ & $4(4.9 \%)$ & 0.576 & 0.961 & $0(0.0 \%)$ & $1(1.7 \%)$ & 1.000 & 1.000 \\
\hline Anti-SRP & $0(0.0 \%)$ & $4(4.9 \%)$ & 0.576 & 0.961 & $0(0.0 \%)$ & $3(5.2 \%)$ & 0.557 & 1.000 \\
\hline Anti-NXP2 & $0(0.0 \%)$ & $9(11.0 \%)$ & 0.199 & 0.816 & $0(0.0 \%)$ & $4(6.9 \%)$ & 0.571 & 1.000 \\
\hline Anti-PM-Scl75 & $3(13.6 \%)$ & $7(8.5 \%)$ & 0.438 & 0.961 & $3(13.6 \%)$ & $5(8.6 \%)$ & 0.677 & 1.000 \\
\hline Anti-PM-Scl100 & $1(4.5 \%)$ & $1(1.2 \%)$ & 0.380 & 0.961 & $1(4.5 \%)$ & $0(0.0 \%)$ & 0.275 & 1.000 \\
\hline Anti-Mi-2a & $1(4.5 \%)$ & $5(6.1 \%)$ & 1.000 & 1.000 & $1(4.5 \%)$ & $3(5.2 \%)$ & 1.000 & 1.000 \\
\hline Anti-Mi-2 $\beta$ & $1(4.5 \%)$ & $4(4.9 \%)$ & 1.000 & 1.000 & $1(4.5 \%)$ & $2(3.4 \%)$ & 1.000 & 1.000 \\
\hline Anti-SAE1 & $1(4.5 \%)$ & $3(3.7 \%)$ & 1.000 & 1.000 & $1(4.5 \%)$ & $3(5.2 \%)$ & 1.000 & 1.000 \\
\hline \multicolumn{9}{|c|}{ Comorbidities/Harmful hobbies } \\
\hline Smoking & $6(27.3 \%)$ & $10(12.2 \%)$ & 0.100 & 0.816 & $6(27.3 \%)$ & $6(10.3 \%)$ & 0.080 & 1.000 \\
\hline Alcohol abuse & $5(22.7 \%)$ & $19(23.2 \%)$ & 0.965 & 1.000 & $5(22.7 \%)$ & $12(20.7 \%)$ & 1.000 & 1.000 \\
\hline Hypertension & $6(27.3 \%)$ & $12(14.6 \%)$ & 0.204 & 0.816 & $6(27.3 \%)$ & $6(10.3 \%)$ & 0.080 & 1.000 \\
\hline Diabetes & $4(18.2 \%)$ & $15(18.3 \%)$ & 1.000 & 1.000 & $4(18.2 \%)$ & $12(20.7 \%)$ & 1.000 & 1.000 \\
\hline Hepatitis & $4(18.2 \%)$ & $17(20.7 \%)$ & 1.000 & 1.000 & $4(18.2 \%)$ & $14(24.1 \%)$ & 0.766 & 1.000 \\
\hline Allergic History & $4(18.2 \%)$ & $12(14.6 \%)$ & 0.741 & 1.000 & $4(18.2 \%)$ & $10(17.2 \%)$ & 1.000 & 1.000 \\
\hline \multicolumn{9}{|l|}{ Therapies } \\
\hline Steroid monotherapy & $4(18.2 \%)$ & $21(25.6 \%)$ & 0.469 & 0.961 & $4(18.2 \%)$ & $14(24.1 \%)$ & 0.766 & 1.000 \\
\hline Steroid + DMARDs & $15(68.2 \%)$ & $42(51.2 \%)$ & 0.156 & 0.816 & $15(68.2 \%)$ & $31(53.4 \%)$ & 0.234 & 1.000 \\
\hline Steroid + IVIG & $2(9.1 \%)$ & $11(13.4 \%)$ & 0.731 & 1.000 & $2(9.1 \%)$ & $7(12.1 \%)$ & 1.000 & 1.000 \\
\hline $\begin{array}{l}\text { Steroid + DMARDs + } \\
\text { IVIG }\end{array}$ & $1(4.5 \%)$ & $8(9.8 \%)$ & 0.680 & 1.000 & $1(4.5 \%)$ & $6(10.3 \%)$ & 0.667 & 1.000 \\
\hline
\end{tabular}

RP-ILD: Rapid progression of interstitial lung disease; IIM-ILD: Idiopathic-inflammatory-myopathy-related interstitial lung disease; y: years; m: months; UIP pattern: Usual interstitial pneumonia pattern; MYOACT: Myositis Disease Activity Assessment Visual Analogue Scales; FVC\%: Percent-predicted forced vital capacity; TLC: Total lung capacity; L: Litre; FEV1\%: Percent-predicted forced expiratory volume in 1 second; FEV1/FVC: Ratio of FEV1 over FVC; DLCO\%: Percent-predicted diffusing capacity of the lung for carbon monoxide; DMARDs: Disease-modifying anti-rheumatic drugs; IVIG: Intravenous immunoglobulin; IIM: Idiopathic inflammatory myopathy; DM: dermatomyositis; PM: Polymyositis; ADM: Amyopathic dermatomyositis. 


\begin{tabular}{|c|c|c|c|c|c|c|c|c|}
\hline \multirow[b]{2}{*}{$\begin{array}{l}\text { Maximum dosage of } \\
\text { steroid }\end{array}$} & \multicolumn{4}{|c|}{ Before propensity score matching } & \multicolumn{4}{|c|}{ After propensity score matching } \\
\hline & $40.0(24.0,80.0)$ & $60.0(40.0,80.0)$ & 0.146 & 0.816 & $40.0(24.0,80.0)$ & $\begin{array}{l}40.0(39.0 \\
80.0)\end{array}$ & 0.263 & 1.000 \\
\hline \multicolumn{9}{|l|}{ IIM subtypes } \\
\hline DM & $16(72.7 \%)$ & $51(62.2 \%)$ & 0.360 & 0.961 & $16(72.7 \%)$ & $35(60.3 \%)$ & 0.304 & 1.000 \\
\hline PM & $4(18.2 \%)$ & $20(24.4 \%)$ & 0.539 & 0.961 & $4(18.2 \%)$ & $15(25.9 \%)$ & 0.471 & 1.000 \\
\hline ADM & $2(9.1 \%)$ & $11(13.4 \%)$ & 0.731 & 1.000 & $2(9.1 \%)$ & $8(13.8 \%)$ & 0.719 & 1.000 \\
\hline \multicolumn{9}{|c|}{$\begin{array}{l}\text { RP-ILD: Rapid progression of interstitial lung disease; IIM-ILD: Idiopathic-inflammatory-myopathy-related interstitial lung disease; y: years; } \\
\text { m: months; UIP pattern: Usual interstitial pneumonia pattern; MYOACT: Myositis Disease Activity Assessment Visual Analogue Scales; } \\
\text { FVC\%: Percent-predicted forced vital capacity; TLC: Total lung capacity; L: Litre; FEV1\%: Percent-predicted forced expiratory volume in } 1 \\
\text { second; FEV1/FVC: Ratio of FEV1 over FVC; DLCO\%: Percent-predicted diffusing capacity of the lung for carbon monoxide; DMARDs: } \\
\text { Disease-modifying anti-rheumatic drugs; IVIG: Intravenous immunoglobulin; IIM: Idiopathic inflammatory myopathy; DM: dermatomyositis } \\
\text { PM: Polymyositis; ADM: Amyopathic dermatomyositis. }\end{array}$} \\
\hline
\end{tabular}

In the setting of time to death from any cause, the univariate Cox proportional hazards regression analysis identified pulmonary bacterial infection $(P=0.035)$, RP-ILD $(P=0.077)$, MYOACT score $(P=0.023)$, positivity of anti-MDA5 antibody $(P=0.003)$ and nintedanib therapy $(P=$ 0.062 ) as factors related to survival in follow-up at the level of $P<0.1$. The subsequent multivariate analysis demonstrated that nintedanib therapy improved the survival of IIM-ILD patients $(P=0.040$, HR value $=0.219)$ after adjusting for other clinical factors. Besides, elevated MYOACT score $(P=0.018)$ and positivity of anti-MDA5 antibody $(P=0.002)$ were found to be risk factors for death in IIM-ILD patients. After propensity score matching, MYOACT score $(P=0.007)$, positivity of anti-MDA5 antibody $(P=0.004)$ and nintedanib therapy $(P=0.027, H R$ value $=0.190)$ remain statistically significant factors for death in these patients (Table 3 ) 
Table 3

Univariate and multivariate Cox proportional hazards regression analysis of survival in IIM-ILD patients

\begin{tabular}{|c|c|c|c|c|c|c|c|c|}
\hline \multirow[b]{2}{*}{ Factors } & \multicolumn{4}{|c|}{ Before propensity score matching } & \multicolumn{4}{|c|}{ After propensity score matching } \\
\hline & $\begin{array}{l}P \\
\text { value }\end{array}$ & $\begin{array}{l}\text { OR } \\
\text { value }\end{array}$ & $95 \% \mathrm{Cl}$ & $\begin{array}{l}\text { P- } \\
\text { adjusted }\end{array}$ & $\begin{array}{l}P \\
\text { value }\end{array}$ & $\begin{array}{l}\text { OR } \\
\text { value }\end{array}$ & $95 \% \mathrm{Cl}$ & $\begin{array}{l}\text { P- } \\
\text { adjusted }\end{array}$ \\
\hline \multicolumn{9}{|l|}{ Univariate analysis } \\
\hline $\operatorname{Age}(y)$ & 0.219 & 1.029 & $0.983 \sim 1.077$ & 0.813 & 0.127 & 1.040 & $0.989 \sim 1.094$ & 1.000 \\
\hline Sex(male/female) & 0.380 & 1.568 & $0.575 \sim 4.274$ & 0.999 & 0.757 & 1.185 & $0.404 \sim 3.473$ & 1.000 \\
\hline Course of disease(m) & 0.965 & 0.999 & $0.972 \sim 1.027$ & 0.999 & 0.777 & 0.996 & $0.968 \sim 1.025$ & 1.000 \\
\hline $\begin{array}{l}\text { Duration of diagnosis } \\
\text { delay(m) }\end{array}$ & 0.159 & 1.039 & $0.985 \sim 1.096$ & 0.813 & 0.256 & 1.032 & $0.978 \sim 1.089$ & 1.000 \\
\hline \multicolumn{9}{|c|}{ Clinical manifestations or complications } \\
\hline Dysphagia & 0.998 & $<0.001$ & $0.000 \sim$ & 0.999 & 0.999 & $<0.001$ & $0.000 \sim$ & 1.000 \\
\hline Dysarthria & 0.999 & $<0.001$ & $0.000 \sim$ & 0.999 & 0.999 & $<0.001$ & $0.000 \sim$ & 1.000 \\
\hline $\begin{array}{l}\text { Respiratory muscle } \\
\text { involvement }\end{array}$ & 0.999 & $<0.001$ & $0.000 \sim$ & 0.999 & 0.999 & $<0.001$ & $0.000 \sim$ & 1.000 \\
\hline Cardiac involvement & 0.999 & $<0.001$ & $0.000 \sim$ & 0.999 & 0.999 & $<0.001$ & $0.000 \sim$ & 1.000 \\
\hline Gastrointestinal hemorrhage & 0.999 & $<0.001$ & $0.000 \sim$ & 0.999 & 0.999 & $<0.001$ & $0.000 \sim$ & 1.000 \\
\hline Pulmonary bacterial infection & 0.884 & 1.088 & $0.350 \sim 3.382$ & 0.999 & 0.538 & 1.458 & $0.439 \sim 4.844$ & 1.000 \\
\hline Pulmonary fungal infection & 0.999 & $<0.001$ & $0.000 \sim$ & 0.999 & 1.000 & $<0.001$ & $0.000 \sim$ & 1.000 \\
\hline Carcinoma & 0.541 & 1.477 & $0.423 \sim 5.161$ & 0.999 & 0.364 & 1.846 & $0.491 \sim 6.941$ & 1.000 \\
\hline UIP pattern & 0.817 & 1.154 & $0.344 \sim 3.875$ & 0.999 & 0.593 & 1.403 & $0.405 \sim 4.865$ & 1.000 \\
\hline Pneumomediastinum & 0.219 & 3.216 & $0.499 \sim 20.737$ & 0.813 & 0.178 & 4.067 & $0.529 \sim 31.267$ & 1.000 \\
\hline \multicolumn{9}{|l|}{ Disease activity } \\
\hline MYOACT score & 0.038 & 1.192 & $1.010 \sim 1.406$ & 0.813 & 0.050 & 1.187 & $1.000 \sim 1.409$ & 0.867 \\
\hline \multicolumn{9}{|l|}{ Lung function testing } \\
\hline FVC\%(\%) & 0.360 & 1.013 & $0.985 \sim 1.043$ & 0.999 & 0.147 & 1.024 & $0.992 \sim 1.057$ & 1.000 \\
\hline $\operatorname{TLC}(\mathrm{L})$ & 0.113 & 0.641 & $0.370 \sim 1.111$ & 0.813 & 0.255 & 0.714 & $0.400 \sim 1.275$ & 1.000 \\
\hline FEV1\%(\%) & 0.637 & 0.993 & $0.967 \sim 1.021$ & 0.999 & 0.692 & 1.006 & $0.976 \sim 1.038$ & 1.000 \\
\hline FEV1/FVC & 0.106 & 0.016 & $0.000 \sim 2.412$ & 0.813 & 0.310 & 0.060 & $0.000 \sim 13.560$ & 1.000 \\
\hline DLCO\%(\%) & 0.198 & 0.981 & $0.952 \sim 1.010$ & 0.813 & 0.583 & 0.989 & $0.952 \sim 1.028$ & 1.000 \\
\hline \multicolumn{9}{|c|}{ Myositis-specific antibodies \& Myositis-associated antibodies } \\
\hline Anti-MDA5 & 0.224 & 1.990 & $0.656 \sim 6.038$ & 0.813 & 0.280 & 1.970 & $0.576 \sim 6.735$ & 1.000 \\
\hline Anti-PL-7 & 0.524 & 1.583 & $0.385 \sim 6.508$ & 0.999 & 0.731 & 1.286 & $0.307 \sim 5.388$ & 1.000 \\
\hline Anti-PL-12 & 0.505 & 2.306 & $0.198 \sim 26.823$ & 0.999 & 1.000 & $<0.001$ & $0.000 \sim$ & 1.000 \\
\hline Anti-EJ & 0.471 & 1.882 & $0.337 \sim 10.526$ & 0.999 & 0.622 & 1.547 & $0.273 \sim 8.771$ & 1.000 \\
\hline Anti-Ro-52 & 0.223 & 1.872 & $0.684 \sim 5.124$ & 0.813 & 0.245 & 1.905 & $0.642 \sim 5.649$ & 1.000 \\
\hline Anti-Jo-1 & 0.452 & 1.622 & $0.460 \sim 5.723$ & 0.999 & 0.571 & 1.455 & $0.398 \sim 5.315$ & 1.000 \\
\hline
\end{tabular}

IIM-ILD: Idiopathic-inflammatory-myopathy-related interstitial lung disease; y: years; m: months; UIP pattern: Usual interstitial pneumonia pattern; RP-ILD: Rapid progression of interstitial lung disease; MYOACT: Myositis Disease Activity Assessment Visual Analogue Scales; FVC\%: Percent-predicted forced vital capacity; TLC: Total lung capacity; L: Litre; FEV1\%: Percent-predicted forced expiratory volume in 1 second; FEV1/FVC: Ratio of FEV1 over FVC; DLCO\%: Percent-predicted diffusing capacity of the lung for carbon monoxide; DMARDs: Disease-modifying anti-rheumatic drugs; IVIG: Intravenous immunoglobulin; IIM: Idiopathic inflammatory myopathy; DM: dermatomyositis; PM: Polymyositis; ADM: Amyopathic dermatomyositis. 


\begin{tabular}{|c|c|c|c|c|c|c|c|c|}
\hline \multirow[b]{2}{*}{ Anti-OJ } & \multicolumn{4}{|c|}{ Before propensity score matching } & \multicolumn{4}{|c|}{ After propensity score matching } \\
\hline & 0.505 & 2.306 & $0.198 \sim 26.823$ & 0.999 & 0.347 & 3.875 & $0.230 \sim 65.386$ & 1.000 \\
\hline Anti-TIF1Y & 0.418 & 0.417 & $0.050 \sim 3.468$ & 0.999 & 0.531 & 0.500 & $0.057 \sim 4.369$ & 1.000 \\
\hline Anti-Ku & 0.999 & $<0.001$ & $0.000 \sim$ & 0.999 & 1.000 & $<0.001$ & $0.000 \sim$ & 1.000 \\
\hline Anti-SRP & 0.999 & $<0.001$ & $0.000 \sim$ & 0.999 & 0.999 & $<0.001$ & $0.000 \sim$ & 1.000 \\
\hline Anti-NXP2 & 0.999 & $<0.001$ & $0.000 \sim$ & 0.999 & 0.999 & $<0.001$ & $0.000 \sim$ & 1.000 \\
\hline Anti-PM-Scl75 & 0.075 & 3.511 & $0.883 \sim 13.961$ & 0.813 & 0.050 & 4.538 & $1.002 \sim 20.553$ & 0.867 \\
\hline Anti-PM-Scl100 & 0.999 & $<0.001$ & $0.000 \sim$ & 0.999 & 1.000 & $<0.001$ & $0.000 \sim$ & 1.000 \\
\hline Anti-Mi-2a & 0.917 & 0.889 & $0.098 \sim 8.079$ & 0.999 & 0.851 & 1.250 & $0.122 \sim 12.840$ & 1.000 \\
\hline Anti-Mi-2 $\beta$ & 0.918 & 1.125 & $0.119 \sim 10.674$ & 0.999 & 0.608 & 1.906 & $0.162 \sim 22.374$ & 1.000 \\
\hline Anti-SAE1 & 0.999 & $<0.001$ & $0.000 \sim$ & 0.999 & 0.999 & $<0.001$ & $0.000 \sim$ & 1.000 \\
\hline \multicolumn{9}{|l|}{ Comorbidities/Harmful hobbies } \\
\hline Smoking & 0.957 & 1.038 & $0.265 \sim 4.076$ & 0.999 & 0.731 & 1.286 & $0.307 \sim 5.388$ & 1.000 \\
\hline Alcohol abuse & 0.817 & 0.867 & $0.258 \sim 2.911$ & 0.999 & 0.796 & 1.183 & $0.330 \sim 4.239$ & 1.000 \\
\hline Hypertension & 0.847 & 0.875 & $0.226 \sim 3.387$ & 0.999 & 0.731 & 1.286 & $0.307 \sim 5.388$ & 1.000 \\
\hline Diabetes & 0.729 & 1.244 & $0.362 \sim 4.283$ & 0.999 & 0.682 & 1.308 & $0.362 \sim 4.727$ & 1.000 \\
\hline Hepatitis & 0.918 & 1.067 & $0.314 \sim 3.629$ & 0.999 & 0.909 & 1.077 & $0.303 \sim 3.828$ & 1.000 \\
\hline Allergic History & 0.205 & 0.259 & $0.032 \sim 2.095$ & 0.813 & 0.186 & 0.240 & $0.029 \sim 1.984$ & 1.000 \\
\hline \multicolumn{9}{|l|}{ Therapies } \\
\hline Steroid monotherapy & 0.797 & 1.161 & $0.372 \sim 3.620$ & 0.999 & 0.444 & 1.603 & $0.479 \sim 5.367$ & 1.000 \\
\hline Steroid + DMARDs & 0.833 & 0.898 & $0.332 \sim 2.434$ & 0.999 & 0.669 & 0.791 & $0.269 \sim 2.319$ & 1.000 \\
\hline Steroid + IVIG & 0.222 & 2.252 & $0.613 \sim 8.275$ & 0.813 & 0.354 & 2.036 & $0,452 \sim 9.162$ & 1.000 \\
\hline Steroid + DMARDs + IVIG & 0.999 & $<0.001$ & $0.000 \sim$ & 0.999 & 0.999 & $<0.001$ & $0.000 \sim$ & 1.000 \\
\hline Maximum dosage of steroid & 0.579 & 1.001 & $0.997 \sim 1.005$ & 0.999 & 0.771 & 1.001 & $0.997 \sim 1.004$ & 1.000 \\
\hline Nintedanib & 0.093 & 0.169 & $0.021 \sim 1.346$ & 0.813 & 0.051 & 0.125 & $0.016 \sim 1.008$ & 0.867 \\
\hline \multicolumn{9}{|l|}{ IIM subtypes } \\
\hline DM & 0.239 & 0.546 & $0.199 \sim 1.495$ & 0.813 & 0.634 & 0.767 & $0.256 \sim 2.294$ & 1.000 \\
\hline PM & 0.711 & 1.241 & $0.396 \sim 3.885$ & 0.999 & 0.981 & 0.985 & $0.279 \sim 3.477$ & 1.000 \\
\hline ADM & 0.222 & 2.252 & $0.613 \sim 8.275$ & 0.813 & 0.474 & 1.714 & $0.393 \sim 7.485$ & 1.000 \\
\hline \multicolumn{9}{|l|}{ Multivariate analysis } \\
\hline MYOACT score & 0.023 & 1.228 & $1.028 \sim 1.468$ & & 0.032 & 1.235 & $1.019 \sim 1.498$ & \\
\hline Anti-PM-Scl75 & 0.044 & 5.025 & $1.045 \sim 24.162$ & & 0.027 & 8.673 & $1.276 \sim 58.957$ & \\
\hline \multirow[t]{2}{*}{ Nintedanib } & 0.048 & 0.107 & $0.012 \sim 0.978$ & & 0.023 & 0.063 & $0.006 \sim 0.681$ & \\
\hline & \multicolumn{4}{|c|}{ Before propensity score matching } & \multicolumn{4}{|c|}{ After propensity score matching } \\
\hline Factors & $\begin{array}{l}P \\
\text { value }\end{array}$ & $\begin{array}{l}\text { HR } \\
\text { value }\end{array}$ & $95 \% \mathrm{Cl}$ & $\begin{array}{l}\text { P- } \\
\text { adjusted }\end{array}$ & $\begin{array}{l}P \\
\text { value }\end{array}$ & $\begin{array}{l}\text { HR } \\
\text { value }\end{array}$ & $95 \% \mathrm{Cl}$ & $\begin{array}{l}\text { P- } \\
\text { adjusted }\end{array}$ \\
\hline $\begin{array}{l}\text { IIM-ILD: Idiopathic-inflammat } \\
\text { pattern; RP-ILD: Rapid progres } \\
\text { FVC\%: Percent-predicted force } \\
\text { second; FEV1/FVC: Ratio of F } \\
\text { Disease-modifying anti-rheum } \\
\text { PM: Polymyositis; ADM: Amy }\end{array}$ & 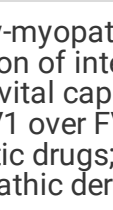 & $\begin{array}{l}\text { ity; TLC } \\
\text { igLCO Intr } \\
\text { itomyc }\end{array}$ & $\begin{array}{l}\text { rstitial lung dise } \\
\text { isease; MYOACT } \\
\text { al lung capacity } \\
\text { ercent-predicted } \\
\text { ous immunoglo }\end{array}$ & $\begin{array}{l}\text { se; y: year } \\
\text { Myositis } \\
\text { L: Litre; FE } \\
\text { iffusing c } \\
\text { ulin; IIM: I }\end{array}$ & $\begin{array}{l}\text { n: mont } \\
\text { ease Ac } \\
\text { \%: Perc } \\
\text { acity of } \\
\text { pathic ir }\end{array}$ & $\begin{array}{l}\text { UIP pat } \\
\text { tiy Asse } \\
\text {-predict } \\
\text { lung fo } \\
\text { immato }\end{array}$ & $\begin{array}{l}\text { : Usual interstitial } \\
\text { ent Visual Analog } \\
\text { orced expiratory v } \\
\text { rbon monoxide; D } \\
\text { nyopathy; DM: der }\end{array}$ & $\begin{array}{l}\text { umonia } \\
\text { cales; } \\
\text { le in } 1 \\
\text { Ds: } \\
\text { myositis; }\end{array}$ \\
\hline
\end{tabular}


Univariate analysis

\begin{tabular}{|c|c|c|c|c|c|c|c|c|}
\hline $\operatorname{Age}(\mathrm{y})$ & 0.156 & 1.026 & $0.990 \sim 1.064$ & 0.624 & 0.584 & 1.011 & $0.972 \sim 1.051$ & 0.986 \\
\hline Sex(male/female) & 0.410 & 1.383 & $0.640 \sim 2.992$ & 0.829 & 0.592 & 1.271 & $0.529 \sim 3.055$ & 0.986 \\
\hline Course of disease(m) & 0.148 & 0.956 & $0.900 \sim 1.016$ & 0.624 & 0.205 & 0.966 & $0.917 \sim 1.019$ & 0.820 \\
\hline $\begin{array}{l}\text { Duration of diagnosis } \\
\text { delay }(m)\end{array}$ & 0.103 & 0.887 & $0.767 \sim 1.025$ & 0.596 & 0.138 & 0.899 & $0.781 \sim 1.035$ & 0.784 \\
\hline \multicolumn{9}{|c|}{ Clinical manifestations or complications } \\
\hline Dysphagia & 0.375 & 1.556 & $0.586 \sim 4.128$ & 0.829 & 0.954 & 1.045 & $0.241 \sim 4.529$ & 0.986 \\
\hline Dysarthria & 0.448 & 0.461 & $0.062 \sim 3.410$ & 0.829 & 0.906 & 0.886 & $0.118 \sim 6.655$ & 0.986 \\
\hline $\begin{array}{l}\text { Respiratory muscle } \\
\text { involvement }\end{array}$ & 0.342 & 2.019 & $0.474 \sim 8.594$ & 0.829 & 0.156 & 2.903 & $0.667 \sim 12.631$ & 0.784 \\
\hline Cardiac involvement & 0.942 & 0.929 & $0.126 \sim 6.870$ & 0.963 & 0.967 & 0.958 & $0.128 \sim 7.185$ & 0.986 \\
\hline Gastrointestinal hemorrhage & 0.202 & 2.008 & $0.687 \sim 5.867$ & 0.750 & 0.537 & 1.476 & $0.429 \sim 5.078$ & 0.986 \\
\hline Pulmonary bacterial infection & 0.035 & 2.326 & $1.060 \sim 5.103$ & 0.596 & 0.082 & 2.224 & $0.903 \sim 5.477$ & 0.784 \\
\hline Pulmonary fungal infection & 0.497 & 0.047 & $\begin{array}{l}0.000 \sim \\
321.715\end{array}$ & 0.829 & 0.719 & 0.048 & $\begin{array}{l}0.000 \sim \\
685997.402\end{array}$ & 0.986 \\
\hline Carcinoma & 0.938 & 0.959 & $0.330 \sim 2.783$ & 0.963 & 0.882 & 0.911 & $0.267 \sim 3.111$ & 0.986 \\
\hline UIP pattem & 0.112 & 2.657 & $0.796 \sim 8.866$ & 0.596 & 0.127 & 2.602 & $0.761 \sim 8.902$ & 0.784 \\
\hline Pneumomediastinum & 0.915 & 0.897 & $0.121 \sim 6.660$ & 0.963 & 0.810 & 1.282 & $0.170 \sim 9.675$ & 0.986 \\
\hline RP-ILD & 0.077 & 2.207 & $0.917 \sim 5.311$ & 0.596 & 0.109 & 2.222 & $0.838 \sim 5.892$ & 0.784 \\
\hline \multicolumn{9}{|l|}{ Disease activity } \\
\hline MYOACT score & 0.023 & 1.148 & $1.019 \sim 1.292$ & 0.596 & 0.006 & 1.198 & $1.052 \sim 1.364$ & 0.208 \\
\hline \multicolumn{9}{|l|}{ Lung function testing } \\
\hline FVC\%(\%) & 0.644 & 0.995 & $0.974 \sim 1.016$ & 0.863 & 0.323 & 1.013 & $0.988 \sim 1.038$ & 0.933 \\
\hline TLC(L) & 0.477 & 0.861 & $0.570 \sim 1.301$ & 0.829 & 0.960 & 0.988 & $0.622 \sim 1.570$ & 0.986 \\
\hline FEV1\%(\%) & 0.353 & 0.990 & $0.970 \sim 1.011$ & 0.829 & 0.393 & 1.011 & $0.986 \sim 1.037$ & 0.986 \\
\hline FEV1/FVC & 0.752 & 0.559 & $0.015 \sim 20.646$ & 0.909 & 0.534 & 4.938 & $0.032 \sim 757.986$ & 0.986 \\
\hline DLCO\%(\%) & 0.338 & 0.989 & $0.966 \sim 1.012$ & 0.829 & 0.425 & 0.987 & $0.955 \sim 1.020$ & 0.986 \\
\hline \multicolumn{9}{|c|}{ Myositis-specific antibodies \& Myositis-associated antibodies } \\
\hline Anti-MDA5 & 0.003 & 3.371 & $1.500 \sim 7.578$ & 0.156 & 0.008 & 3.663 & $1.413 \sim 9.500$ & 0.208 \\
\hline Anti-PL-7 & 0.944 & 0.958 & $0.287 \sim 3.191$ & 0.963 & 0.995 & 0.996 & $0.291 \sim 3.402$ & 0.995 \\
\hline Anti-PL-12 & 0.570 & 1.787 & $0.241 \sim 13.256$ & 0.863 & 0.719 & 0.048 & $\begin{array}{l}0.000 \sim \\
685997.402\end{array}$ & 0.986 \\
\hline Anti-EJ & 0.486 & 0.491 & $0.066 \sim 3.633$ & 0.829 & 0.495 & 0.496 & $0.066 \sim 3.721$ & 0.986 \\
\hline Anti-Ro-52 & 0.276 & 1.535 & $0.710 \sim 3.320$ & 0.829 & 0.689 & 1.196 & $0.498 \sim 2.875$ & 0.986 \\
\hline Anti-Jo-1 & 0.664 & 0.788 & $0.269 \sim 2.311$ & 0.863 & 0.743 & 0.830 & $0.273 \sim 2.526$ & 0.986 \\
\hline
\end{tabular}

IIM-ILD: Idiopathic-inflammatory-myopathy-related interstitial lung disease; y: years; m: months; UIP pattern: Usual interstitial pneumonia pattern; RP-ILD: Rapid progression of interstitial lung disease; MYOACT: Myositis Disease Activity Assessment Visual Analogue Scales; FVC\%: Percent-predicted forced vital capacity; TLC: Total lung capacity; L: Litre; FEV1\%: Percent-predicted forced expiratory volume in 1 second; FEV1/FVC: Ratio of FEV1 over FVC; DLCO\%: Percent-predicted diffusing capacity of the lung for carbon monoxide; DMARDs:

Disease-modifying anti-rheumatic drugs; IVIG: Intravenous immunoglobulin; IIM: Idiopathic inflammatory myopathy; DM: dermatomyositis; PM: Polymyositis; ADM: Amyopathic dermatomyositis. 


\begin{tabular}{|c|c|c|c|c|c|c|c|c|}
\hline \multirow[b]{2}{*}{ Anti-OJ } & \multicolumn{4}{|c|}{ Before propensity score matching } & \multicolumn{4}{|c|}{ After propensity score matching } \\
\hline & 0.478 & 2.071 & $0.278 \sim 15.444$ & 0.829 & 0.666 & 0.048 & $\begin{array}{l}0.000 \sim \\
48534.441\end{array}$ & 0.986 \\
\hline Anti-TIF1Y & 0.983 & 1.013 & $0.304 \sim 3.379$ & 0.983 & 0.809 & 0.835 & $0.193 \sim 3.604$ & 0.986 \\
\hline Anti-Ku & 0.501 & 0.047 & $\begin{array}{l}0.000 \sim \\
352.610\end{array}$ & 0.829 & 0.719 & 0.048 & $\begin{array}{l}0.000 \sim 102 \\
685997.402\end{array}$ & 0.986 \\
\hline Anti-SRP & 0.500 & 0.047 & $\begin{array}{l}0.000 \sim \\
344.901\end{array}$ & 0.829 & 0.565 & 0.047 & $0.000 \sim 1600.979$ & 0.986 \\
\hline Anti-NXP2 & 0.353 & 0.388 & $0.052 \sim 2.864$ & 0.829 & 0.858 & 1.203 & $0.159 \sim 9.126$ & 0.986 \\
\hline Anti-PM-Scl75 & 0.782 & 0.815 & $0.192 \sim 3.463$ & 0.924 & 0.843 & 1.160 & $0.267 \sim 5.042$ & 0.986 \\
\hline Anti-PM-Scl100 & 0.510 & 1.995 & $0.256 \sim 15.567$ & 0.829 & 0.628 & 0.044 & $\begin{array}{l}0.000 \sim \\
13295.545\end{array}$ & 0.986 \\
\hline Anti-Mi-2a & 0.725 & 0.698 & $0.094 \sim 5.116$ & 0.909 & 0.896 & 1.144 & $0.153 \sim 8.576$ & 0.986 \\
\hline Anti-Mi-2 $\beta$ & 0.927 & 0.911 & $0.123 \sim 6.760$ & 0.963 & 0.562 & 1.819 & $0.241 \sim 13.748$ & 0.986 \\
\hline Anti-SAE1 & 0.886 & 0.864 & $0.116 \sim 6.430$ & 0.963 & 0.904 & 0.883 & $0.117 \sim 6.675$ & 0.986 \\
\hline \multicolumn{9}{|l|}{ Comorbidities/Harmful hobbies } \\
\hline Smoking & 0.429 & 1.486 & $0.557 \sim 3.966$ & 0.829 & 0.914 & 1.070 & $0.312 \sim 3.677$ & 0.986 \\
\hline Alcohol abuse & 0.592 & 1.270 & $0.530 \sim 3.045$ & 0.863 & 0.263 & 1.742 & $0.659 \sim 4.609$ & 0.855 \\
\hline Hypertension & 0.637 & 0.773 & $0.265 \sim 2.251$ & 0.863 & 0.550 & 0.640 & $0.148 \sim 2.722$ & 0.986 \\
\hline Diabetes & 0.623 & 0.765 & $0.263 \sim 2.224$ & 0.863 & 0.719 & 0.797 & $0.232 \sim 2.741$ & 0.986 \\
\hline Hepatitis & 0.747 & 1.163 & $0.466 \sim 2.901$ & 0.909 & 0.286 & 1.696 & $0.642 \sim 4.481$ & 0.875 \\
\hline Allergic History & 0.102 & 0.189 & $0.026 \sim 1.396$ & 0.596 & 0.150 & 0.228 & $0.030 \sim 1.708$ & 0.784 \\
\hline \multicolumn{9}{|l|}{ Therapies } \\
\hline Steroid monotherapy & 0.230 & 0.520 & $0.179 \sim 1.513$ & 0.797 & 0.140 & 0.333 & $0.077 \sim 1.437$ & 0.784 \\
\hline Steroid + DMARDs & 0.474 & 0.755 & $0.350 \sim 1.630$ & 0.829 & 0.891 & 1.065 & $0.435 \sim 2.607$ & 0.986 \\
\hline Steroid + IVIG & 0.116 & 2.195 & $0.823 \sim 5.860$ & 0.596 & 0.399 & 1.701 & $0.495 \sim 5.847$ & 0.986 \\
\hline Steroid + DMARDs + IVIG & 0.126 & 2.309 & $0.790 \sim 6.746$ & 0.596 & 0.181 & 2.328 & $0.676 \sim 8.022$ & 0.784 \\
\hline Maximum dosage of steroid & 0.104 & 1.001 & $1.000 \sim 1.003$ & 0.596 & 0.179 & 1.001 & $0.999 \sim 1.003$ & 0.784 \\
\hline Nintedanib & 0.062 & 0.252 & $0.059 \sim 1.072$ & 0.596 & 0.054 & 0.237 & $0.054 \sim 1.026$ & 0.784 \\
\hline \multicolumn{9}{|l|}{ IIM subtypes } \\
\hline DM & 0.652 & 1.211 & $0.526 \sim 2.787$ & 0.863 & 0.249 & 1.815 & $0.658 \sim 5.004$ & 0.855 \\
\hline PM & 0.560 & 0.748 & $0.281 \sim 1.989$ & 0.863 & 0.222 & 0.464 & $0.135 \sim 1.594$ & 0.825 \\
\hline ADM & 0.913 & 1.070 & $0.318 \sim 3.597$ & 0.963 & 0.966 & 0.969 & $0.222 \sim 4.224$ & 0.986 \\
\hline \multicolumn{9}{|l|}{ Multivariate analysis } \\
\hline MYOACT score & 0.018 & 1.155 & $1.025 \sim 1.303$ & & 0.007 & 1.197 & $1.051 \sim 1.364$ & \\
\hline Anti-MDA5 & 0.002 & 3.598 & $1.596 \sim 8.110$ & & 0.004 & 4.249 & $1.584 \sim 11.396$ & \\
\hline Nintedanib & 0.040 & 0.219 & $0.051 \sim 0.931$ & & 0.027 & 0.190 & $0.043 \sim 0.827$ & \\
\hline \multicolumn{9}{|c|}{$\begin{array}{l}\text { IIM-ILD: Idiopathic-inflammatory-myopathy-related interstitial lung disease; y: years; m: months; UIP pattern: Usual interstitial pneumonia } \\
\text { pattern; RP-ILD: Rapid progression of interstitial lung disease; MYOACT: Myositis Disease Activity Assessment Visual Analogue Scales; } \\
\text { FVC\%: Percent-predicted forced vital capacity; TLC: Total lung capacity; L: Litre; FEV1\%: Percent-predicted forced expiratory volume in } 1 \\
\text { second; FEV1/FVC: Ratio of FEV1 over FVC; DLCO\%: Percent-predicted diffusing capacity of the lung for carbon monoxide; DMARDs: } \\
\text { Disease-modifying anti-rheumatic drugs; IVIG: Intravenous immunoglobulin; IIM: Idiopathic inflammatory myopathy; DM: dermatomyositis } \\
\text { PM: Polymyositis; ADM: Amyopathic dermatomyositis. }\end{array}$} \\
\hline
\end{tabular}


In the 22 patients receiving compassionate nintedanib therapy, nintedanib was initially administered 150 mg twice daily as per the manufacturer's recommendation. The dosage was reduced to $100 \mathrm{mg}$ twice daily due to adverse events in 6 patients. And 6 patients finally terminated nintedanib therapy (4 due to intolerable adverse events and 2 due to mortality). Dosage reduction or therapy discontinuation occurred to $45.5 \%$ of nintedanib-treated pateints. During a mean nintedanib therapy period of $12.8 \pm 7.1$ months, adverse events occurred to $63.6 \%$ of patients, and diarrhea was the most common adverse event (54.5\%), followed by fatigue (18.2\%) and cough (18.2\%). Although hepatic insufficiency only happened to 3 patients (13.6\%), it was the major adverse event directly associated with dosage reduction (50\%) or therapy discontinuation (50\%) in the follow-up interviews. (Fig. 4, Supplementary table 2)

\section{Discussion}

Nintedanib is an intracellular inhibitor that targets multiple tyrosine kinases and hereby inhibits fundamental processes in the pathogenesis of fibrosis. Although proven to be effective in IPF and Ssc-related ILD, the efficacy and tolerability of nintedanib in other connective-tissue-diseaserelated ILD (CTD-ILD), IIM-ILD in particular, has never been clarified. To date, this is the first clinical research focusing on nintedanib therapy in IIM-ILD patients. And nintedanib was found effective in reducing incidence of RP-ILD and improving survival in these patients. However, patients receiving nintedanib therapy did not suffer from less complication of pulmonary infection or receive more simplified immunosuppressive regimen as we expected. Tolerability of nintedanib therapy was as well evaluated by recording and discussing the adverse events, dosage reduction and discontinuation of the therapy.

Nintedanib has already been proved of therapeutic value in reducing lung function decline and acute exacerbation of idiopathic pulmonary fibrosis (AE-IPF) in IPF patients. The previous TOMORROW trial, INPULSIS-1 and INPULSIS-2 trials demonstrated that nintedanib promisingly reduced the annual rate of decline in FVC $[16,17]$. The therapeutic effect of nintedanib remain consistent irrespective of age, sex, ethnicity and baseline percent-predicted forced vital capacity (FVC\%) $[28,29]$. And the efficacy in slowing lung function decline could last over 3 years [30]. Similar results were also reported in several real-world experience across Europe during the Compassionate Use Programs (CUPs) or after license [31-33]. In addition, the TOMORROW trial and INPULSIS-2 trial also demonstrated that nintedanib played a protective role in preventing AE-IPF [34]. In INPULSIS-2 trial, nintedanib significantly increased the time to the first acute exacerbation. Meanwhile the incidence of acute exacerbation was reduced in TOMORROW trial. The following INPULSIS-ON trial also suggested that nintedanib's therapeutic effect in reducing occurrence of acute exacerbation persisted beyond 3 years. In in SENSCIS trial, nintedanib was identified to decrease FVC decline in Ssc-ILD, nevertheless, its effect on RP-ILD was not probed into [18]. The recently completed INBUILD trial demonstrated the consistent effect of nintedanib on reducing FVC decline in different ILD subgroups, irrespective of the underlying ILD diagnosis [20]. In our study, nintedanib was found to reduce the incidence of the fatal RP-ILD in IIM-ILD patients, which for the first time initially demonstrated the therapeutic value of nintedanib in IIM-ILD regardless of the retrospective nature and lack of follow-up lung function testing. In the future, large-cohort,prospective, multicenter research concerning IIM-ILD is demanded to verify the role of nintedanib in decreasing incidence of RP-ILD, as well as explore its effect in lung function decline, etc.

In addition to decreasing the incidence of RP-ILD in follow-up, nintedanib was also observed to benefit the survival of IIM-ILD patients. Similar finding in IPF patients was also reported in an Italian multicenter study with a favorable 1-year survival of 79\% [35]. On the one hand, RP-ILD has been proven to be a risk factor for death in IIM-ILD patients $[10,11]$. Nintedanib might improve IIM patients' survival by reducing the incidence of RP-ILD. On the other hand, nintedanib was still found to play a protective role in survival after adjusting for RP-ILD, etc., which led us to consider the possible therapeutic value of nintedanib beyond ILD. In preclinical models of systemic sclerosis, nintedanib was confirmed to alleviate skin fibrosis by decreasing of dermal thickness, myofibroblast counts and hydroxyproline content [36]. However, no clinical benefit of nintedanib was identified for extra-pulmonary manifestations of systemic sclerosis in SENSCIS trial [18]. In terms of IIM, preceding studies proposed the essential role of tyrosine kinase in type I IFN signaling in pathogenesis of DM [37]. As a tyrosine kinase inhibitor [38], the effect of nintedanib on disease activity, muscular or extra-muscular involvements as well as pathogenesis of IIM, DM in particular, is worth exploration in the future.

During the course of nintedanib therapy, adverse events were inevitable in most patients and might impede the therapeutic regimen by dosage reduction or discontinuation of therapy. In our study, $63.6 \%$ of IIM-ILD patients receiving nintedanib therapy suffered from adverse events. Almost half of nintedanib-treated patients finally received reduced dosage of nintedanib or discontinued the therapy. The incidence of adverse events was much lower than those (>90\%) reported in preceding clinical trials possibly owing to exclusion of IPF progression, upper respiratory tract infection, etc. from adverse events [16-18]. Diarrhea was the most common adverse event in IIM-ILD patients, which resembled the reports from series of clinical trials and real-world analyses [16-18, 20, 31-33, 39]. Besides, liver function monitoring and timely liver-protected medication should be emphasized in IIM-ILD patients since it was identified as the major direct contributor to dosage reduction or discontinuation due to adverse event.

MSAs and MAAs are two main subtypes of antibodies in IIM patients. They were valuable in the field of diagnosis and evaluation. In preceding studies, anti-MDA5 antibody was found to be correlated with ILD, RP-ILD as well as death in DM or ADM patients [40-42]. However, its role in

Page $13 / 23$ 
ILD and progression of ILD remained disputable [43]. In this study, anti-MDA5 antibody was recognized as a risk factor for death in IIM-ILD patients. Nevertheless, no correlation was identified between anti-MDA5 antibody and RP-ILD, which indicated the unreliable role of anti-MDA5 antibody in predicting progression of ILD. In addition, anti PM-Scl75 antibody, which was previously taken as an inconspicuous MSA indicating risk of overlapping with systemic sclerosis, was found to be associated with RP-ILD in IIM-ILD patients. Lorenzo, etc. proposed that anti-PM-Sclpositive patients tended to exhibit more extra-muscular features like ILD, esophageal reflux disease, etc. in comparison with other myositis patients [44]. Preceding evidence on its linkage with RP-ILD was not sufficient. In a French multicenter study, only $17 \%$ of myositis patients with anti-PM-Scl antibody suffered from deterioration of ILD [45]. In contrast, Lega and his colleagues found that ILD worsened in 4 out of 9 anti-PMScl-positive patients [46]. This is the first study suggesting the predicting role of anti-PM-Scl75 antibody in RP-ILD among IIM-ILD patients. In the future, larger cohort study is demanded to verify our finding.

Elevated MYOACT score, which reflected higher disease activity in IIM patients, was found to be correlated with RP-ILD and death. As a core set measure for IIM patients, MYOACT score work as an overall assessment of IIM disease activity by taking muscular and various extra-muscular manifestations into consideration. It has been demonstrated to predict outcome of IIM patients and reflect inflammatory level [47, 48]. Meanwhile RP-ILD was also found to be associated with cytokines like tumor necrosis factor, etc [49]. The partially overlapped pathological mechanism might make MYOACT score a satisfying predictor for RP-ILD in IIM-ILD patients.

The most significant limitations of this study were the retrospective and observational nature as well as the small sample size. Due to the initial lack of follow-up lung function testing and recent impact of COVID-19 pandemic, we failed to clarify the effect of nintedanib on lung function decline. Baseline FVC\%, DLCO\%, age, immunosuppressive therapy, etc. were not controlled for inclusion because of the limited number of IIMILD patients receiving nintedanib therapy. No placebo was given to patients in the control group. Alterations of life quality, MYOACT score, muscle strength, etc. after nintedanib therapy were not recorded. Last but not least, underestimation of RP-ILD might occur in patients under conventional medications owing to initial lack of mandatory management. In spite of all the limitations, we intended to initially figure out the therapeutic value of nintedanib in IIM-ILD, and shed some light on the future therapeutic landscape of CTD-ILD.

\section{Conclusion}

In IIM-ILD patients, nintedanib might be effective in reducing incidence of RP-ILD as well as improving survival. The efficacy of nintedanib in lung function decline, life quality and disease activity of IIM, etc. demands exploration in future study. Besides, anti-MDA5 antibody was verified to be a risk factor for death in IIM-ILD patients, and anti-PM-Scl75 antibody might be valuable in predicting occurrence of RP-ILD. Among IIMILD patients receiving nintedanib therapy, diarrhea was the most common adverse event. Hepatic insufficiency frequently led to dosage reduction or therapy discontinuation.

\section{Abbreviations}

IIM

Idiopathic inflammatory myopathy;

DM

Dermatomyositis;

PM

Polymyositis;

ADM

Amyopathic dermatomyositis;

ILD

Interstitial lung disease;

IIM-ILD

Idiopathic-inflammatory-myopathy-related interstitial lung disease;

RP-ILD

Rapid progression of interstitial lung disease;

VEGF

Vascular endothelial growth factor;

PDGF

Platelet-derived growth factor;

FGF

Fibroblast growth factor;

IPF

Idiopathic pulmonary fibrosis; 
SSC-ILD

Systemic-sclerosis-related interstitial lung disease;

RA-ILD

Rheumatoid-arthritis-related interstitial lung disease;

FAHZJU

The First Affiliated Hospital, College of Medicine, Zhejiang University;

CTD

Connective tissue disease;

EMR

Electronic medical record;

MYOACT

Myositis Disease Activity Assessment Visual Analogue Scales;

HRCT

High-resolution computed tomography;

UIP

Usual interstitial pneumonia;

ICD-10

International Statistical Classification of Diseases, 10th revision

CAP

Community-acquired pneumonia;

HAP

Hospital-acquired pneumonia;

BALF

Bronchoalveolar lavage fluid;

MSA

Myositis-specific antibody;

MAA

Myositits-associated antibody;

COVID-19

Corona virus disease 2019;

FVC

Forced vital capacity;

FDR

False discovery rate;

DLCO\%

Percent-predicted diffusing capacity of the lung for carbon monoxide;

CTD-ILD

connective-tissue-disease-related interstitial lung disease;

AE-IPF

Acute exacerbation of idiopathic pulmonary fibrosis;

FVC\%

Percent-predicted forced vital capacity

CUP

Compassionate Use Progrpam

\section{Declarations}

\section{Ethics approval and consent to participate}

The study was approved (2020-200, 2018-224) by the Clinical Research Ethics Committee of FAHZJU and retrospectively registered at ISRCTN.com, ISRCTN 10507540. All participants gave written informed consent.

\section{Consent for publication}

Consents for publication from 22 patients receiving nintedanib therapy were also acquired prior to submission. 
Part of the datasets can be seen in the supplements of this work. Other datasets used and/or analyzed during the current study are available from the corresponding author on reasonable request.

\section{Competing interests}

The authors declare that they have no competing interests.

\section{Funding}

This study was supported by the grants from National Natural Science Foundation of China (81701602) and Natural Science Foundation of Zhejiang Province (LQ20H100003).

\section{Authors' contributions}

Junyu Liang contributed to the study design, data collection, statistical analysis, writing and proof reading of this work. Heng Cao contributed to the study design, data collection and proof reading of this study. Yang Yang, Yini Ke, Chuanyin Sun and Lihuan Yue contributed to the data collection and proof reading of the manuscript. Jin Lin contributed to the study design and proof reading of this study. All authors read and approved the final manuscript.

\section{Acknowledgements}

Not applicable.

\section{References}

1. Hoogendijk JE, Amato AA, Lecky BR, Choy EH, Lundberg IE, Rose MR, Vencovsky J, de Visser M, Hughes RA: 119th ENMC international workshop: trial design in adult idiopathic inflammatory myopathies, with the exception of inclusion body myositis, 10-12 October 2003, Naarden, The Netherlands. Neuromuscul Disord 2004, 14(5):337-345.

2. Prieto S, Grau JM. The geoepidemiology of autoimmune muscle disease. Autoimmun Rev. 2010;9(5):A330-4.

3. Mammen AL. Autoimmune myopathies: autoantibodies, phenotypes and pathogenesis. Nat Rev Neurol. 2011;7(6):343-54.

4. Li L, Wang H, Wang Q, Wu C, Liu C, Zhang Y, Cheng L, Zeng X, Zhang F, Li Y. Myositis-specific autoantibodies in dermatomyositis/polymyositis with interstitial lung disease. J Neurol Sci. 2019;397:123-8.

5. Fathi M, Vikgren J, Boijsen M, Tylen U, Jorfeldt L, Tornling G, Lundberg IE. Interstitial lung disease in polymyositis and dermatomyositis: longitudinal evaluation by pulmonary function and radiology. Arthritis Rheum. 2008;59(5):677-85.

6. Fathi M, Dastmalchi M, Rasmussen E, Lundberg IE, Tornling G. Interstitial lung disease, a common manifestation of newly diagnosed polymyositis and dermatomyositis. Ann Rheum Dis. 2004;63(3):297-301.

7. Saketkoo LA, Ascherman DP, Cottin V, Christopher-Stine L, Danoff SK, Oddis CV. Interstitial Lung Disease in Idiopathic Inflammatory Myopathy. Curr Rheumatol Rev. 2010;6(2):108-19.

8. Sugiyama Y, Yoshimi R, Tamura M, Takeno M, Kunishita Y, Kishimoto D, Yoshioka Y, Kobayashi K, Takase-Minegishi K, Watanabe T, et al. The predictive prognostic factors for polymyositis/dermatomyositis-associated interstitial lung disease. Arthritis Res Ther. 2018;20(1):7.

9. Ning Y, Yang G, Sun Y, Chen S, Liu Y, Shi G. Efficiency of Therapeutic Plasma-Exchange in Acute Interstitial Lung Disease, Associated With Polymyositis/Dermatomyositis Resistant to Glucocorticoids and Immunosuppressive Drugs: A Retrospective Study. Front Med (Lausanne). 2019;6:239.

10. Ishikawa Y, Iwata S, Hanami K, Nawata A, Zhang M, Yamagata K, Hirata S, Sakata K, Todoroki Y, Nakano K, et al. Relevance of interferongamma in pathogenesis of life-threatening rapidly progressive interstitial lung disease in patients with dermatomyositis. Arthritis Res Ther. 2018;20(1):240.

11. Lian X, Zou J, Guo Q, Chen S, Lu L, Wang R, Zhou M, Fu Q, Ye Y, Bao C. Mortality Risk Prediction in Amyopathic Dermatomyositis Associated With Interstitial Lung Disease. Chest: The FLAIR Model; 2020.

12. Hilberg F, Roth GJ, Krssak M, Kautschitsch S, Sommergruber W, Tontsch-Grunt U, Garin-Chesa P, Bader G, Zoephel A, Quant J, et al. BIBF 1120: triple angiokinase inhibitor with sustained receptor blockade and good antitumor efficacy. Cancer Res. 2008;68(12):4774-82.

13. Wollin L, Maillet I, Quesniaux V, Holweg A, Ryffel B. Antifibrotic and anti-inflammatory activity of the tyrosine kinase inhibitor nintedanib in experimental models of lung fibrosis. J Pharmacol Exp Ther. 2014;349(2):209-20. 
14. Bendstrup E, Wuyts W, Alfaro T, Chaudhuri N, Cornelissen R, Kreuter M, Melgaard Nielsen K, Munster AB, Myllarniemi M, Ravaglia C, et al. Nintedanib in Idiopathic Pulmonary Fibrosis: Practical Management Recommendations for Potential Adverse Events. Respiration. 2019;97(2):173-84.

15. Wollin L, Distler JHW, Redente EF, Riches DWH, Stowasser S, Schlenker-Herceg R, Maher TM, Kolb M. Potential of nintedanib in treatment of progressive fibrosing interstitial lung diseases. Eur Respir J 2019, 54(3).

16. Richeldi L, Costabel U, Selman M, Kim DS, Hansell DM, Nicholson AG, Brown KK, Flaherty KR, Noble PW, Raghu G, et al. Efficacy of a tyrosine kinase inhibitor in idiopathic pulmonary fibrosis. N Engl J Med. 2011;365(12):1079-87.

17. Richeldi L, du Bois RM, Raghu G, Azuma A, Brown KK, Costabel U, Cottin V, Flaherty KR, Hansell DM, Inoue Y, et al. Efficacy and safety of nintedanib in idiopathic pulmonary fibrosis. N Engl J Med. 2014;370(22):2071-82.

18. Distler O, Highland KB, Gahlemann M, Azuma A, Fischer A, Mayes MD, Raghu G, Sauter W, Girard M, Alves M, et al. Nintedanib for Systemic Sclerosis-Associated Interstitial Lung Disease. N Engl J Med. 2019;380(26):2518-28.

19. Rivera-Ortega P, Hayton C, Blaikley J, Leonard C, Chaudhuri N. Nintedanib in the management of idiopathic pulmonary fibrosis: clinical trial evidence and real-world experience. Ther Adv Respir Dis. 2018;12:1753466618800618.

20. Wells AU, Flaherty KR, Brown KK, Inoue Y, Devaraj A, Richeldi L, Moua T, Crestani B, Wuyts WA, Stowasser S, et al. Nintedanib in patients with progressive fibrosing interstitial lung diseases-subgroup analyses by interstitial lung disease diagnosis in the INBUILD trial: a randomised, double-blind, placebo-controlled, parallel-group trial. Lancet Respir Med. 2020;8(5):453-60.

21. Lundberg IE, Tjarnlund A, Bottai M, Werth VP, Pilkington C, Visser M, Alfredsson L, Amato AA, Barohn RJ, Liang MH, et al. 2017 European League Against Rheumatism/American College of Rheumatology classification criteria for adult and juvenile idiopathic inflammatory myopathies and their major subgroups. Ann Rheum Dis. 2017;76(12):1955-64.

22. Isenberg DA, Allen E, Farewell V, Ehrenstein MR, Hanna MG, Lundberg IE, Oddis C, Pilkington C, Plotz P, Scott D, et al. International consensus outcome measures for patients with idiopathic inflammatory myopathies. Development and initial validation of myositis activity and damage indices in patients with adult onset disease. Rheumatology. 2004;43(1):49-54.

23. Sato T, Teramukai S, Kondo H, Watanabe A, Ebina M, Kishi K, Fujii Y, Mitsudomi T, Yoshimura M, Maniwa T, et al. Impact and predictors of acute exacerbation of interstitial lung diseases after pulmonary resection for lung cancer. J Thorac Cardiovasc Surg. 2014;147(5):1604-11 e1603.

24. Hozumi H, Fujisawa T, Nakashima R, Johkoh T, Sumikawa H, Murakami A, Enomoto N, Inui N, Nakamura Y, Hosono Y, et al. Comprehensive assessment of myositis-specific autoantibodies in polymyositis/dermatomyositis-associated interstitial lung disease. Respir Med. 2016;121:91-9.

25. Horiike Y, Suzuki Y, Fujisawa T, Yasui H, Karayama M, Hozumi H, Furuhashi K, Enomoto N, Nakamura Y, Inui N, et al. Successful classification of macrophage-mannose receptor CD206 in severity of anti-MDA5 antibody positive dermatomyositis associated ILD. Rheumatology. 2019;58(12):2143-52.

26. Sato S, Hirakata M, Kuwana M, Suwa A, Inada S, Mimori T, Nishikawa T, Oddis CV, Ikeda Y. Autoantibodies to a 140-kd polypeptide, CADM140, in Japanese patients with clinically amyopathic dermatomyositis. Arthritis Rheum. 2005;52(5):1571-6.

27. Abe Y, Matsushita M, Tada K, Yamaji K, Takasaki Y, Tamura N. Clinical characteristics and change in the antibody titres of patients with anti-MDA5 antibody-positive inflammatory myositis. Rheumatology. 2017;56(9):1492-7.

28. Costabel U, Inoue Y, Richeldi L, Collard HR, Tschoepe I, Stowasser S, Azuma A. Efficacy of Nintedanib in Idiopathic Pulmonary Fibrosis across Prespecified Subgroups in INPULSIS. Am J Respir Crit Care Med. 2016;193(2):178-85.

29. Kolb M, Richeldi L, Behr J, Maher TM, Tang W, Stowasser S, Hallmann C, du Bois RM. Nintedanib in patients with idiopathic pulmonary fibrosis and preserved lung volume. Thorax. 2017;72(4):340-6.

30. Rodriguez-Portal JA. Efficacy and Safety of Nintedanib for the Treatment of Idiopathic Pulmonary Fibrosis: An Update. Drugs R D. 2018;18(1):19-25.

31. Bonella F, Kreuter M, Hagmeyer L, Neurohr C, Keller C, Kohlhaeufl MJ, Muller-Quernheim J, Milger K, Prasse A. German Nintedanib Compassionate Use C: Insights from the German Compassionate Use Program of Nintedanib for the Treatment of Idiopathic Pulmonary Fibrosis. Respiration. 2016;92(2):98-106.

32. Toellner H, Hughes G, Beswick W, Crooks MG, Donaldson C, Forrest I, Hart SP, Leonard C, Major M, Simpson AJ, et al. Early clinical experiences with nintedanib in three UK tertiary interstitial lung disease centres. Clin Transl Med. 2017;6(1):41.

33. Brunnemer E, Walscher J, Tenenbaum S, Hausmanns J, Schulze K, Seiter M, Heussel CP, Warth A, Herth FJF, Kreuter M. Real-World Experience with Nintedanib in Patients with Idiopathic Pulmonary Fibrosis. Respiration. 2018;95(5):301-9.

34. Varone F, Sgalla G, lovene B, Bruni T, Richeldi L. Nintedanib for the treatment of idiopathic pulmonary fibrosis. Expert Opin Pharmacother. 2018;19(2):167-75. 
35. Harari S, Caminati A, Poletti V, Confalonieri M, Gasparini S, Lacedonia D, Luppi F, Pesci A, Sebastiani A, Spagnolo P, et al. A Real-Life Multicenter National Study on Nintedanib in Severe Idiopathic Pulmonary Fibrosis. Respiration. 2018;95(6):433-40.

36. Huang J, Beyer C, Palumbo-Zerr K, Zhang Y, Ramming A, Distler A, Gelse K, Distler O, Schett G, Wollin L, et al. Nintedanib inhibits fibroblast activation and ameliorates fibrosis in preclinical models of systemic sclerosis. Ann Rheum Dis. 2016;75(5):883-90.

37. Paniagua RT, Fiorentino DF, Chung L, Robinson WH. Tyrosine kinases in inflammatory dermatologic disease. J Am Acad Dermatol. 2011;65(2):389-403.

38. Liu F, Bayliss G, Zhuang S. Application of nintedanib and other potential anti-fibrotic agents in fibrotic diseases. Clin Sci (Lond). 2019;133(12):1309-20.

39. Tzouvelekis A, Karampitsakos T, Kontou M, Granitsas A, Malliou I, Anagnostopoulos A, Ntolios P, Tzilas V, Bouros E, Steiropoulos P, et al. Safety and efficacy of nintedanib in idiopathic pulmonary fibrosis: A real-life observational study in Greece. Pulm Pharmacol Ther. 2018;49:61-6.

40. Mecoli CA, Christopher-Stine L. Management of Interstitial Lung Disease in Patients With Myositis Specific Autoantibodies. Curr Rheumatol Rep. 2018;20(5):27.

41. Moghadam-Kia S, Oddis CV, Sato S, Kuwana M, Aggarwal R. Antimelanoma Differentiation-associated Gene 5 Antibody: Expanding the Clinical Spectrum in North American Patients with Dermatomyositis. J Rheumatol. 2017;44(3):319-25.

42. Motegi SI, Sekiguchi A, Toki S, Kishi C, Endo Y, Yasuda M, Ikeuchi H, Sakairi T, Hara K, Yamaguchi K, et al. Clinical features and poor prognostic factors of anti-melanoma differentiation-associated gene 5 antibody-positive dermatomyositis with rapid progressive interstitial lung disease. Eur J Dermatol. 2019;29(5):511-7.

43. Hall JC, Casciola-Rosen L, Samedy LA, Werner J, Owoyemi K, Danoff SK, Christopher-Stine L. Anti-melanoma differentiation-associated protein 5-associated dermatomyositis: expanding the clinical spectrum. Arthritis Care Res (Hoboken). 2013;65(8):1307-15.

44. De Lorenzo R, Pinal-Fernandez I, Huang W, Albayda J, Tiniakou E, Johnson C, Milisenda JC, Casal-Dominguez M, Corse AM, Danoff SK, et al. Muscular and extramuscular clinical features of patients with anti-PM/Scl autoantibodies. Neurology. 2018;90(23):e2068-76.

45. Marie I, Lahaxe L, Benveniste O, Delavigne K, Adoue D, Mouthon L, Hachulla E, Constans J, Tiev K, Diot E, et al. Long-term outcome of patients with polymyositis/ dermatomyositis and anti-PM-Scl antibody. Br J Dermatol. 2010;162(2):337-44.

46. Lega JC, Cottin V, Fabien N, Thivolet-Bejui F, Cordier JF. Interstitial lung disease associated with anti-PM/Scl or anti-aminoacyl-tRNA synthetase autoantibodies: a similar condition? J Rheumatol. 2010;37(5):1000-9.

47. Plestilova L, Mann H, Andres Cerezo L, Pecha O, Vencovsky J, Senolt L. The metastasis promoting protein S100A4 levels associate with disease activity rather than cancer development in patients with idiopathic inflammatory myopathies. Arthritis Res Ther. 2014;16(5):468.

48. Rider LG, Aggarwal R, Machado PM, Hogrel JY, Reed AM, Christopher-Stine L, Ruperto N. Update on outcome assessment in myositis. Nat Rev Rheumatol. 2018;14(5):303-18.

49. Kotani T, Takeuchi T, Ishida T, Masutani R, Isoda K, Hata K, Yoshida S, Makino S, Hanafusa T. Increased Serum LIGHT Levels Correlate with Disease Progression and Severity of Interstitial Pneumonia in Patients with Dermatomyositis: A Case Control Study. PLoS One.

2015;10(10):e0140117.

\section{Tables}

\section{Table 1: Unadjusted comparisons between patients receiving nintedanib therapy and patients under conventional medications}

y: years; m: months; UIP pattern: Usual interstitial pneumonia pattern; RP-ILD: Rapid progression of interstitial lung disease; MYOACT: Myositis Disease Activity Assessment Visual Analogue Scales; FVC\%: Percent-predicted forced vital capacity; TLC: Total lung capacity; L: Litre; FEV1\%: Percent-predicted forced expiratory volume in 1 second; FEV1/FVC: Ratio of FEV1 over FVC; DLCO\%: Percent-predicted diffusing capacity of the lung for carbon monoxide; DMARDs: Disease-modifying anti-rheumatic drugs; IVIG: Intravenous immunoglobulin; IIM: Idiopathic inflammatory myopathy; DM: dermatomyositis; PM: Polymyositis; ADM: Amyopathic dermatomyositis.

${ }^{\text {a }}$ medium (interquartile) follow-up time, the log-rank test. 


\begin{tabular}{|c|c|c|c|c|c|c|c|c|}
\hline \multirow[b]{2}{*}{ Factors } & \multicolumn{4}{|c|}{ Before propensity score matching } & \multicolumn{4}{|c|}{ After propensity score matching } \\
\hline & Nintedanib(22) & $\begin{array}{l}\text { Non- } \\
\text { nintedanib(82) }\end{array}$ & $\begin{array}{l}P \\
\text { value }\end{array}$ & $\begin{array}{l}\text { P- } \\
\text { adjusted }\end{array}$ & Nintedanib(22) & $\begin{array}{l}\text { Non } \\
\text { nintedanib(58) }\end{array}$ & $\begin{array}{l}P \\
\text { value }\end{array}$ & $\begin{array}{l}\text { P- } \\
\text { adjusted }\end{array}$ \\
\hline $\operatorname{Age}(y)$ & $55.5(47.3,64.3)$ & $58.5(49.8,65.0)$ & 0.459 & 0.961 & $55.5(47.3,64.3)$ & $57.5(48.5,63.5)$ & 0.678 & 1.000 \\
\hline Sex(male/female) & $9 / 13$ & $31 / 51$ & 0.790 & 1.000 & $9 / 13$ & $26 / 32$ & 0.752 & 1.000 \\
\hline Course of disease(m) & $4.5(2.0,10.3)$ & $3.5(1.0,9.0)$ & 0.500 & 0.961 & $4.5(2.0,10.3)$ & $4.0(1.0,12.5)$ & 0.991 & 1.000 \\
\hline $\begin{array}{l}\text { Duration of diagnosis } \\
\text { delay }(m)\end{array}$ & $2.0(1.0,5.0)$ & $2.5(1.0,5.0)$ & 0.725 & 1.000 & $2.0(1.0,5.0)$ & $3.0(1.0,7.3)$ & 0.713 & 1.000 \\
\hline \multicolumn{9}{|c|}{ Clinical manifestations or complications } \\
\hline Dysphagia & $3(13.6 \%)$ & $12(14.6 \%)$ & 1.000 & 1.000 & $3(13.6 \%)$ & $4(6.9 \%)$ & 0.386 & 1.000 \\
\hline Dysarthria & $1(4.5 \%)$ & $7(8.5 \%)$ & 1.000 & 1.000 & $1(4.5 \%)$ & $3(5.2 \%)$ & 1.000 & 1.000 \\
\hline $\begin{array}{l}\text { Respiratory muscle } \\
\text { involvement }\end{array}$ & $0(0.0 \%)$ & $4(4.9 \%)$ & 0.576 & 0.961 & $0(0.0 \%)$ & $3(5.2 \%)$ & 0.557 & 1.000 \\
\hline Cardiac involvement & $2(9.1 \%)$ & $2(2.4 \%)$ & 0.196 & 0.816 & $2(9.1 \%)$ & $2(3.4 \%)$ & 0.303 & 1.000 \\
\hline $\begin{array}{l}\text { Gastrointestinal } \\
\text { hemomhage }\end{array}$ & $3(13.6 \%)$ & $5(6.1 \%)$ & 0.361 & 0.961 & $3(13.6 \%)$ & $4(6.9 \%)$ & 0.386 & 1.000 \\
\hline $\begin{array}{l}\text { Pulmonary bacterial } \\
\text { infection }\end{array}$ & $4(18.2 \%)$ & $22(26.8 \%)$ & 0.406 & 0.961 & $4(18.2 \%)$ & $15(25.9 \%)$ & 0.471 & 1.000 \\
\hline $\begin{array}{l}\text { Pulmonary fungal } \\
\text { infection }\end{array}$ & $0(0.0 \%)$ & $4(4.9 \%)$ & 0.576 & 0.961 & $0(0.0 \%)$ & $1(1.7 \%)$ & 1.000 & 1.000 \\
\hline Carcinoma & $3(13.6 \%)$ & $14(17.1 \%)$ & 1.000 & 1.000 & $3(13.6 \%)$ & $10(17.2 \%)$ & 1.000 & 1.000 \\
\hline UIP pattern & $6(27.3 \%)$ & $18(22.0 \%)$ & 0.599 & 0.961 & $6(27.3 \%)$ & $17(29.3 \%)$ & 0.857 & 1.000 \\
\hline Pneumomediastinum & $1(4.5 \%)$ & $4(4.9 \%)$ & 1.000 & 1.000 & $1(4.5 \%)$ & $3(5.2 \%)$ & 1.000 & 1.000 \\
\hline Survivala $^{a}(m)$ & $12.8(8.4,19.6)$ & $9.6(6.3,14.2)$ & 0.043 & 0.816 & $12.8(8.4,19.6)$ & $9.8(6.3,14.7)$ & 0.036 & 0.936 \\
\hline RP-ILD & $1(4.5 \%)$ & $18(22.0 \%)$ & 0.069 & 0.816 & $1(4.5 \%)$ & $16(27.6 \%)$ & 0.031 & 0.936 \\
\hline \multicolumn{9}{|l|}{ Disease activity } \\
\hline MYOACT score & $8.0(7.0,9.3)$ & $8.0(6.0,10.0)$ & 0.776 & 1.000 & $8.0(7.0,9.3)$ & $8.0(6.0,10.3)$ & 0.729 & 1.000 \\
\hline \multicolumn{9}{|l|}{ Lung function testing } \\
\hline FVC\%(\%) & $67.2 \pm 16.6$ & $73.7 \pm 18.2$ & 0.134 & 0.816 & $67.2 \pm 16.6$ & $71.5 \pm 18.1$ & 0.333 & 1.000 \\
\hline $\operatorname{TLC}(\mathrm{L})$ & $3.6 \pm 1.2$ & $3.8 \pm 0.9$ & 0.598 & 0.961 & $3.6 \pm 1.2$ & $3.7 \pm 0.9$ & 0.955 & 1.000 \\
\hline FEV1\%(\%) & $69.3 \pm 17.0$ & $75.8 \pm 18.8$ & 0.145 & 0.816 & $69.3 \pm 17.0$ & $72.7 \pm 17.6$ & 0.429 & 1.000 \\
\hline FEV1/FVC & $0.8(0.8,0.9)$ & $0.8(0.8,0.9)$ & 0.759 & 1.000 & $0.8(0.8,0.9)$ & $0.8(0.8,0.9)$ & 0.910 & 1.000 \\
\hline DLCO\%(\%) & $51.7 \pm 13.7$ & $63.4 \pm 18.0$ & 0.006 & 0.312 & $51.7 \pm 13.7$ & $56.1 \pm 14.5$ & 0.220 & 1.000 \\
\hline \multicolumn{9}{|c|}{ Myositis-specific antibodies \& Myositis-associated antibodies } \\
\hline Anti-MDA5 & $6(27.3 \%)$ & $16(19.5 \%)$ & 0.557 & 0.961 & $6(27.3 \%)$ & $10(17.2 \%)$ & 0.355 & 1.000 \\
\hline Anti-PL-7 & $2(9.1 \%)$ & $10(12.2 \%)$ & 1.000 & 1.000 & $2(9.1 \%)$ & $10(17.2 \%)$ & 0.495 & 1.000 \\
\hline Anti-PL-12 & $1(4.5 \%)$ & $2(2.4 \%)$ & 0.514 & 0.961 & $1(4.5 \%)$ & $0(0.0 \%)$ & 0.275 & 1.000 \\
\hline Anti-EJ & $3(13.6 \%)$ & $4(4.9 \%)$ & 0.162 & 0.816 & $3(13.6 \%)$ & $4(6.9 \%)$ & 0.386 & 1.000 \\
\hline Anti-Ro-52 & $11(50.0 \%)$ & $36(43.9 \%)$ & 0.610 & 0.961 & $11(50.0 \%)$ & $26(44.8 \%)$ & 0.679 & 1.000 \\
\hline Anti-Jo-1 & $2(9.1 \%)$ & $14(17.1 \%)$ & 0.513 & 0.961 & $2(9.1 \%)$ & $13(22.4 \%)$ & 0.215 & 1.000 \\
\hline Anti-OJ & $1(4.5 \%)$ & $2(2.4 \%)$ & 0.514 & 0.961 & $1(4.5 \%)$ & $1(1.7 \%)$ & 0.477 & 1.000 \\
\hline Anti-TIF1y & $0(0.0 \%)$ & $11(13.4 \%)$ & 0.115 & 0.816 & $0(0.0 \%)$ & $8(13.8 \%)$ & 0.099 & 1.000 \\
\hline \multirow[t]{2}{*}{ Anti-Ku } & $0(0.0 \%)$ & $4(4.9 \%)$ & 0.576 & 0.961 & $0(0.0 \%)$ & $1(1.7 \%)$ & 1.000 & 1.000 \\
\hline & & & \multicolumn{6}{|c|}{ Page $19 / 23$} \\
\hline
\end{tabular}




\begin{tabular}{|c|c|c|c|c|c|c|c|c|}
\hline Anti-SRP & $0(0.0 \%)$ & $4(4.9 \%)$ & 0.576 & 0.961 & $0(0.0 \%)$ & $3(5.2 \%)$ & 0.557 & 1.000 \\
\hline Anti-NXP2 & $0(0.0 \%)$ & $9(11.0 \%)$ & 0.199 & 0.816 & $0(0.0 \%)$ & $4(6.9 \%)$ & 0.571 & 1.000 \\
\hline Anti-PM-Scl75 & $3(13.6 \%)$ & $7(8.5 \%)$ & 0.438 & 0.961 & $3(13.6 \%)$ & $5(8.6 \%)$ & 0.677 & 1.000 \\
\hline Anti-PM-Scl100 & $1(4.5 \%)$ & $1(1.2 \%)$ & 0.380 & 0.961 & $1(4.5 \%)$ & $0(0.0 \%)$ & 0.275 & 1.000 \\
\hline Anti-Mi-2a & $1(4.5 \%)$ & $5(6.1 \%)$ & 1.000 & 1.000 & $1(4.5 \%)$ & $3(5.2 \%)$ & 1.000 & 1.000 \\
\hline Anti-Mi-2 $\beta$ & $1(4.5 \%)$ & $4(4.9 \%)$ & 1.000 & 1.000 & $1(4.5 \%)$ & 2(3.4\%) & 1.000 & 1.000 \\
\hline Anti-SAE1 & $1(4.5 \%)$ & $3(3.7 \%)$ & 1.000 & 1.000 & $1(4.5 \%)$ & $3(5.2 \%)$ & 1.000 & 1.000 \\
\hline \multicolumn{9}{|c|}{ Comorbidities/Harmful hobbies } \\
\hline Smoking & $6(27.3 \%)$ & $10(12.2 \%)$ & 0.100 & 0.816 & $6(27.3 \%)$ & $6(10.3 \%)$ & 0.080 & 1.000 \\
\hline Alcohol abuse & $5(22.7 \%)$ & $19(23.2 \%)$ & 0.965 & 1.000 & $5(22.7 \%)$ & $12(20.7 \%)$ & 1.000 & 1.000 \\
\hline Hypertension & $6(27.3 \%)$ & $12(14.6 \%)$ & 0.204 & 0.816 & $6(27.3 \%)$ & $6(10.3 \%)$ & 0.080 & 1.000 \\
\hline Diabetes & $4(18.2 \%)$ & $15(18.3 \%)$ & 1.000 & 1.000 & $4(18.2 \%)$ & $12(20.7 \%)$ & 1.000 & 1.000 \\
\hline Hepatitis & $4(18.2 \%)$ & $17(20.7 \%)$ & 1.000 & 1.000 & $4(18.2 \%)$ & $14(24.1 \%)$ & 0.766 & 1.000 \\
\hline Allergic History & $4(18.2 \%)$ & $12(14.6 \%)$ & 0.741 & 1.000 & $4(18.2 \%)$ & $10(17.2 \%)$ & 1.000 & 1.000 \\
\hline \multicolumn{9}{|l|}{ Therapies } \\
\hline Steroid monotherapy & $4(18.2 \%)$ & $21(25.6 \%)$ & 0.469 & 0.961 & $4(18.2 \%)$ & $14(24.1 \%)$ & 0.766 & 1.000 \\
\hline Steroid+DMARDs & $15(68.2 \%)$ & $42(51.2 \%)$ & 0.156 & 0.816 & $15(68.2 \%)$ & $31(53.4 \%)$ & 0.234 & 1.000 \\
\hline Steroid+IVIG & $2(9.1 \%)$ & $11(13.4 \%)$ & 0.731 & 1.000 & $2(9.1 \%)$ & $7(12.1 \%)$ & 1.000 & 1.000 \\
\hline Steroid+DMARDs+IVIG & $1(4.5 \%)$ & $8(9.8 \%)$ & 0.680 & 1.000 & $1(4.5 \%)$ & $6(10.3 \%)$ & 0.667 & 1.000 \\
\hline $\begin{array}{l}\text { Maximum dosage of } \\
\text { steroid }\end{array}$ & $40.0(24.0,80.0)$ & $60.0(40.0,80.0)$ & 0.146 & 0.816 & $40.0(24.0,80.0)$ & $\begin{array}{l}40.0(39.0 \\
80.0)\end{array}$ & 0.263 & 1.000 \\
\hline \multicolumn{9}{|l|}{ IIM subtypes } \\
\hline DM & $16(72.7 \%)$ & $51(62.2 \%)$ & 0.360 & 0.961 & $16(72.7 \%)$ & $35(60.3 \%)$ & 0.304 & 1.000 \\
\hline PM & $4(18.2 \%)$ & $20(24.4 \%)$ & 0.539 & 0.961 & $4(18.2 \%)$ & $15(25.9 \%)$ & 0.471 & 1.000 \\
\hline ADM & $2(9.1 \%)$ & $11(13.4 \%)$ & 0.731 & 1.000 & $2(9.1 \%)$ & $8(13.8 \%)$ & 0.719 & 1.000 \\
\hline
\end{tabular}

\section{Figures}




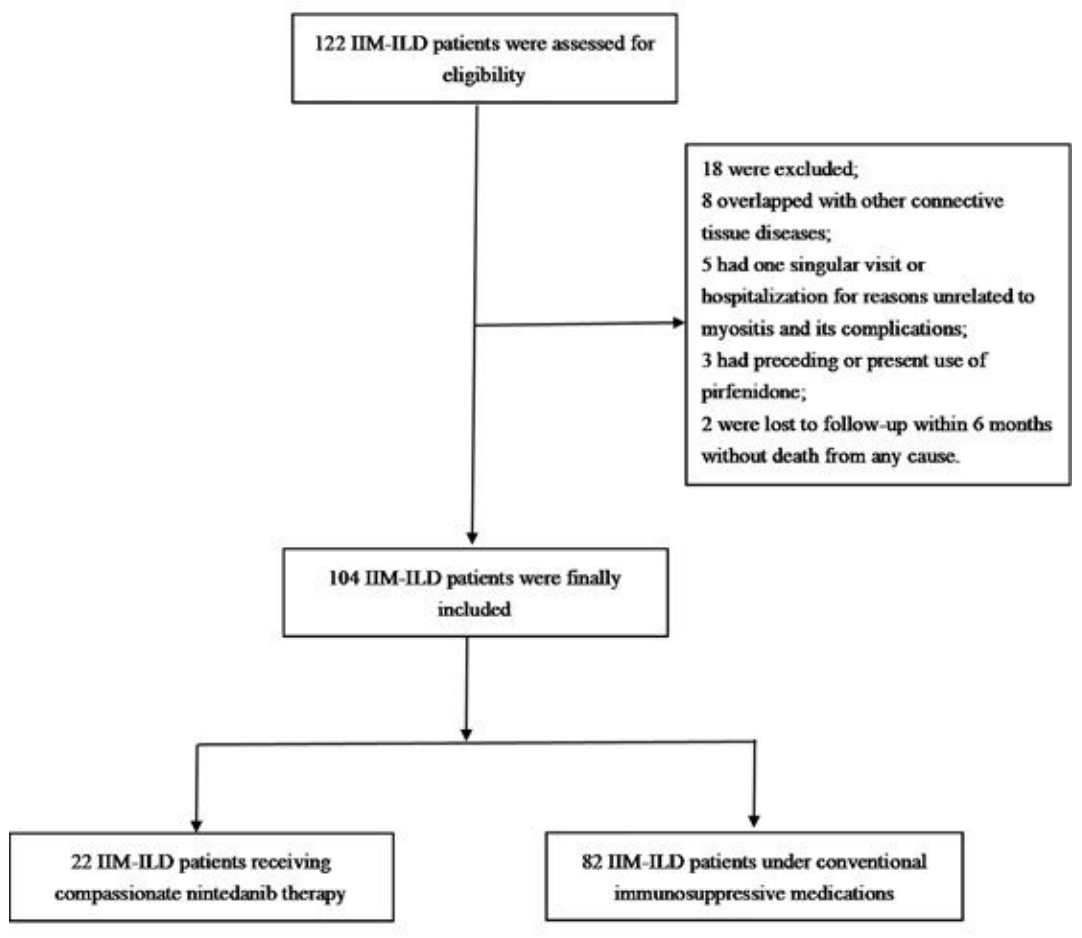

\section{Figure 1}

Enrollment and groupings of this study IIM-ILD: Idiopathic-inflammatory-myopathy-related interstitial lung disease

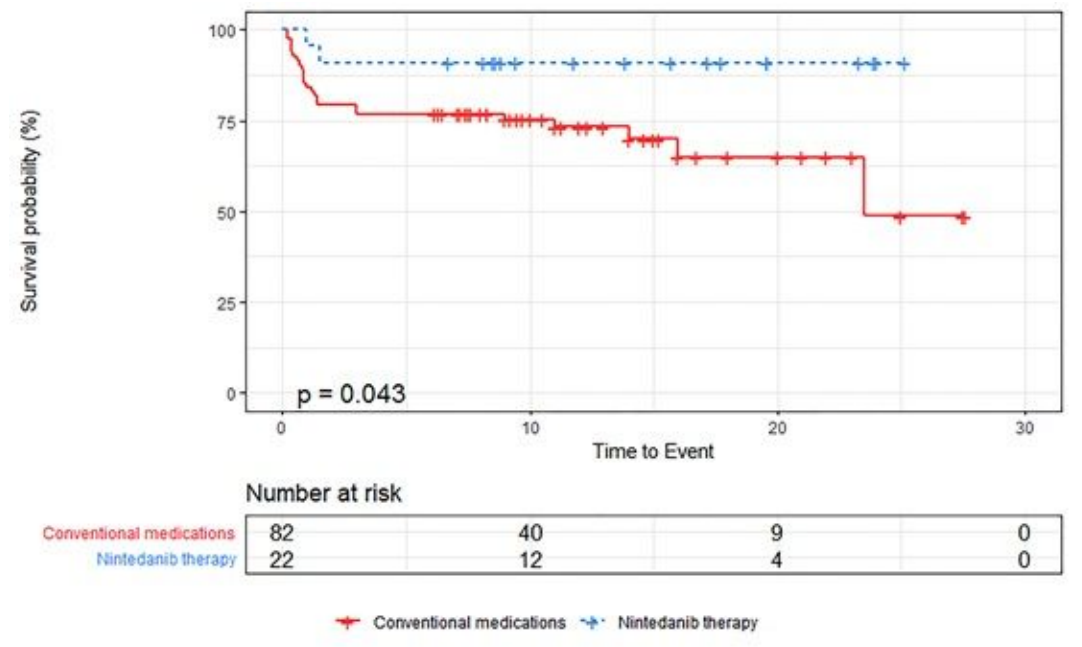

\section{Figure 2}

The Kaplan-Meier curves between patients receiving nintedanib therapy and patients under conventional medications before PSM. PSM Propensity score matching. 


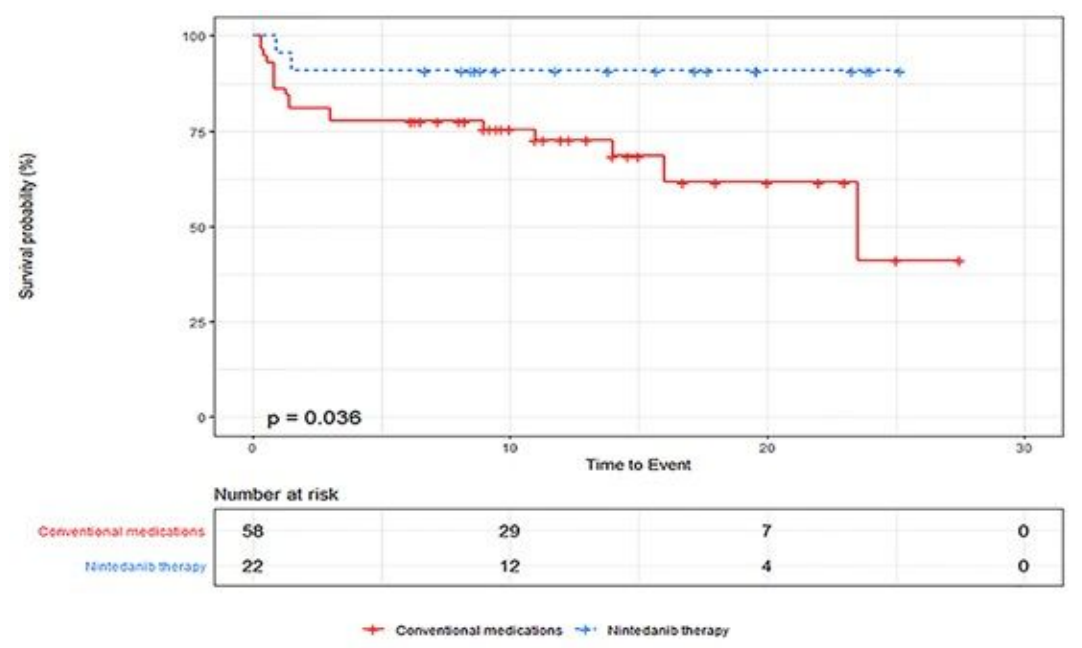

\section{Figure 3}

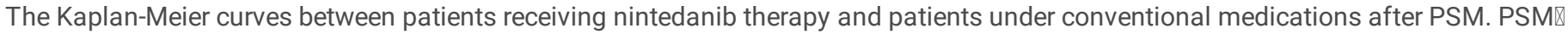
Propensity score matching.

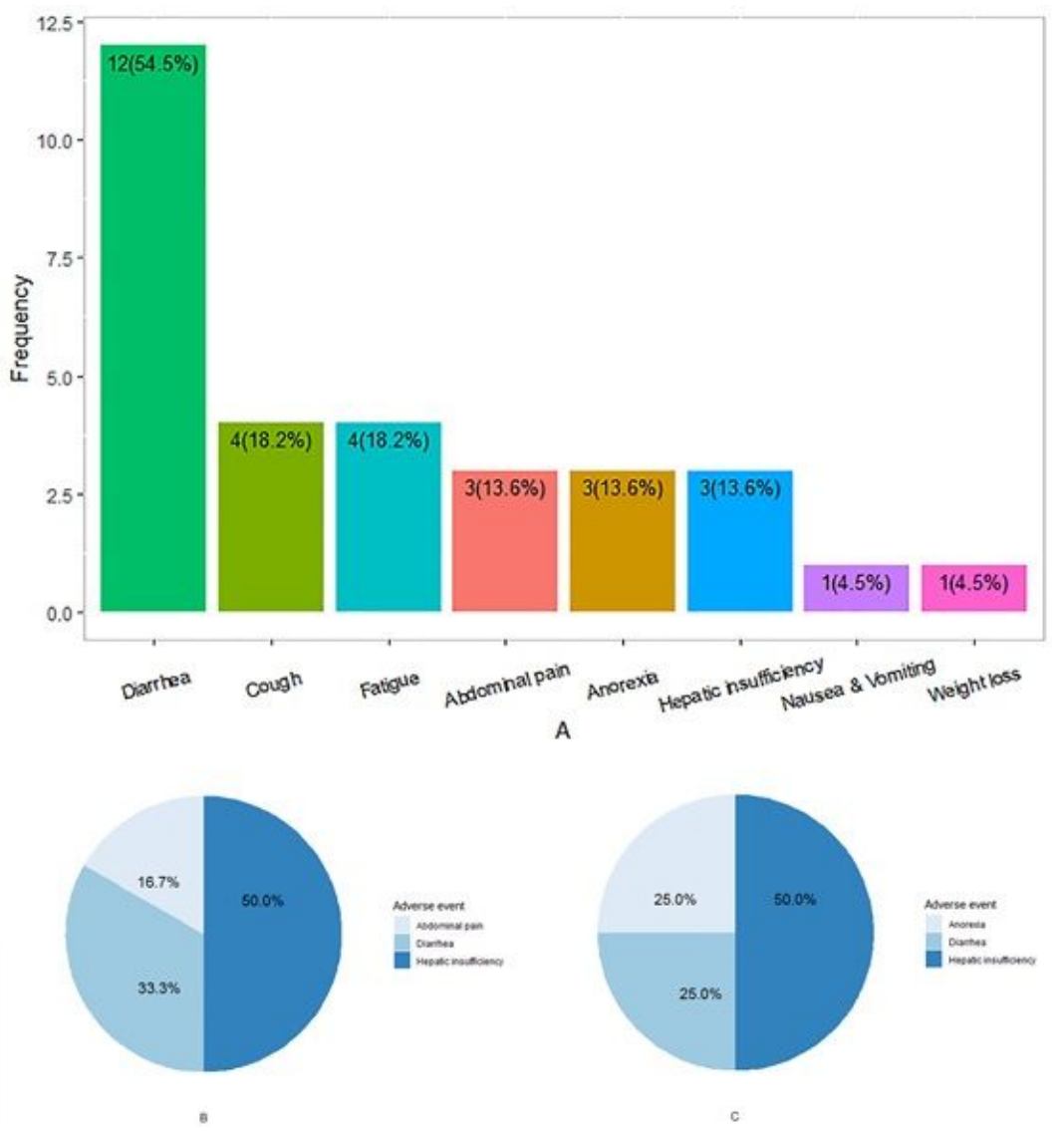

\section{Figure 4}

Adverse events and direct contribution to dosage reduction or therapy discontinuation of nintedanib Panel A showed distribution of adverse events within 22 patients receiving compassionate nintedanib therapy; Panel B revealed distribution of adverse events directly related to dosage reduction of nintedanib in 6 patients; Panel $C$ exhibited distribution of adverse events directly related to therapy discontinuation of nintedanib in 4 patients.

\section{Supplementary Files}

This is a list of supplementary files associated with this preprint. Click to download. 
- Supplementarytable2.docx

- Supplementarytable1.docx

Page 23/23 